\title{
Alternative mixed-integer linear programming models of a maritime
}

\author{
inventory routing problem \\ Jiang Yongheng ${ }^{1,2}$ and Ignacio E. Grossmann ${ }^{3}$ \\ ${ }^{1}$ Institute of Process Control Eng., Department of Automation, Tsinghua University, Beijing, \\ 100084, P.R. China \\ ${ }^{2}$ Tsinghua National Laboratory for Information Science and Technology, Tsinghua University, \\ Beijing 100084, P. R. China \\ ${ }^{3}$ Center for Advanced Process Decision-making, Department of Chemical Engineering, Carnegie \\ Mellon University, Pittsburgh, PA 15217, USA
}

\begin{abstract}
A single product maritime inventory routing problem is addressed in this paper by exploring the use of continuous and discrete time models. We first present a continuous time model based on time slots for single docks, which is enhanced by reformulating the time assignment constraints. Next, we present a model based on event points to handle parallel docks. A discrete time is also presented based on a single commodity fixed-charge network flow problem (FCNF). All the models are solved for multiple randomly generated instances of different problems to compare their computational efficiency, and to illustrate the solutions obtained. .
\end{abstract}

\section{Introduction}

Maritime transportation is a major mode of transportation covering more than $80 \%$ of the world trade by volume (Agra et al, 2013; UNCTAD, 2008). When one actor or cooperating actors in the maritime supply chain have the responsibility of both the transportation of goods and the inventories at the ports, the underlying planning problem is a maritime inventory routing problem (MIRP). Such problems are complex. However, improvements in the fleet utilization and charge/discharge amounts can translate into large cost reductions. This means that there is great potential and need for research in the area of MIRPs (Agra et al, 2013).

Many works related to MIRP have been reported in the literatures. Ronen $(1983,1993)$ published two reviews on ship scheduling and related areas, where different levels and modes were discussed. Christiansen (2004) reviewed the status and perspectives of ship routing and scheduling in 2004. Andersson et al (2010) surveyed the combined inventory management and routing problem from industrial and modeling aspects, and suggested future research with regard to both further development of the research area and industrial needs. Agra et al (2013) reviewed the advances in MIRP, and studied valid inequalities for a single product MIRP to tighten a discrete time model. Surveys by Andersson et al (2010) and Christiansen and Fagerholt (2009) showed that MIRP has received increasing attention in the last decade. As pointed out by Agra et al (2013), the real MIRPs are of high complexity.

There are many works on modeling of MIRPs. Sherali et al (1999) formulated a mixed-integer programming model based on a discrete time representation for the Kuwait Petroleum 
Corporation (KPC) problem, and presented an alternative aggregate model that retains the main features of the KPC problem to improve the computational efficiency. Savelsbergh and Song (2008) developed a discrete time model for the inventory routing problem with continuous moves based on the integer multi-commodity flow formulation. Furman et al (2011) developed a mixed-integer programming formulation based on a discrete time representation for vacuum gas oil routing and inventory management. Song and Furman (2013) introduced a flexible modeling framework for inventory routing problem, which can accommodate various practical features using a discrete time representation. Al-Khayyal and Hwang (2007) studied inventory constrained maritime routing and scheduling for multi- commodity liquid bulk. They defined a position $(i, m)$, where $i$ denotes a port, and $m$ is the arrival number of the vessels at that port within the planning horizon. Then, they formulated the continuous time constraints of different arrivals within one port and different positions related to the same vessel. $\mathrm{Li}$, Karimi and Srinivasan (2010) addressed an inventory service problem in which a chemical MNC uses a fleet of multi-parcel ships with dedicated compartments to move multiple chemicals continuously among its internal and external production and consumption sites. They presented a MILP model similar to that of Al-Khayyal and Hwang (2007) at an operational level with finer granularity. Siswanto (2011) presented a variation of Al-Khayyal and Hwang's model in which he relaxed the problem to consider an assignment of multiundedicated compartments to products. Bilgen and Ozkarahan (2007) presented a MILP model based on a discrete time representation that integrates blending, loading and transportation decisions simultaneously into one model in order to obtain an optimal solution. As pointed out by Sherali and Al-Yakoob (2005), it is possible to solve small practical instances using commercial solvers. However, it is difficult to solve large scale problems due to the large number of binary variables. A computationally efficient model is critical for solving such problems. Furthermore, a discrete time model is generally much larger than continuous time models. Since most of the works have focused on discrete time models, we explore in this paper the potential of continuous time models.

A single product MIRP problem is studied in this paper. There are two categories of ports: production ports and consumption ports. Each port has an inventory with a certain capacity. Vessels transport products from production ports to consumption ports to ensure that the inventories of ports neither exceed given capacities, nor lie below given safety levels. The ports have given production/consumption rates and berth capacities. The vessels have given capacities, speeds, preparation times and charge/discharge rates. The objective is to determine the times that vessels visit the ports, and the charged/discharged amounts of vessels at each port in order to minimize total cost while satisfying the inventory constraints.

From the view point of modeling and solution, MIRP is a complex MILP problem. Model efficiency is a key issue, since different models usually exhibit very different computational requirements. We develop different models for continuous and discrete time and compare them by the sizes, solutions and CPU times in 6 problems. The main difference between the problem in this paper and that in Agra et al's paper (2013) is that in our problem, operations (charges/discharges) require preparation times, and the sailing costs involve a fixed part and a variable part that depends on the loads. 
The remainder of the paper is organized as follows. In section 2, we describe the maritime inventory routing problem. In section 3 , different time presentations are discussed. Then, four models, for continuous time and discrete time, are presented in section 4. The computational results for_multiple randomly generated instances of 6 problems are presented in section 5 . Conclusions are finally given in section 6 .

\section{Problem statement}

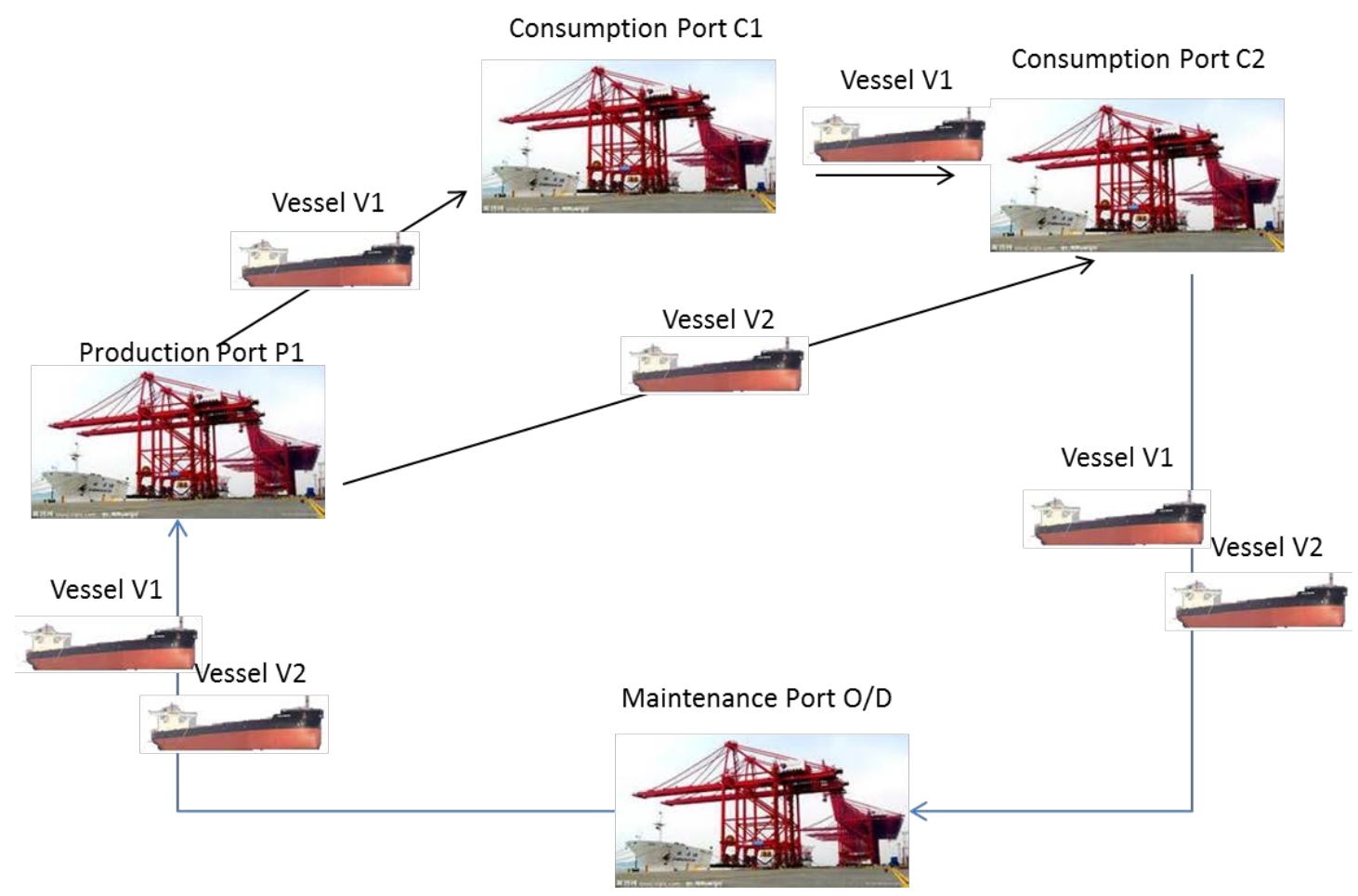

(The pictures of the vessels and ports come from http://www.zhongsou.net and http://www.nipic.com respectively)

Figure 1 Illustrative structure of maritime route

As an illustrative example of the MIRP problem addressed in this paper, we consider as shown in Figure 1, one production port and two consumption ports, which produce or consume products at a certain rate. Vessel V1 departs from the maintenance port (for convenience, we call it here the departure port, denoted as $\mathrm{O}$ ), visits production port $\mathrm{P} 1$ and consumption ports C1, C2 sequentially to transport the product, and finally arrives at the maintenance port (for convenience, we call it here the destination port, denoted as D). Vessel V2 departs from O, visits production port $\mathrm{P} 1$ and consumption ports $\mathrm{C} 2$ sequentially to transport the product, and finally arrives at $D$. The task is to schedule the visiting times and charge/discharge amounts of every vessel to reduce transportation and operation cost with certain constraints. For more general problems, there are more ports and vessels, and different vessels sail by different routes.

The MIRP problem can be stated as follows. 


\section{Given:}

The set of production ports $i \in N^{p}$ and consumption ports $i \in N^{c}$ with production and consumption rates $\mathrm{P}_{i}$ and $\mathrm{Q}_{i}$. The stock of each port must lie between specified lower and upper bounds,

$\mathrm{st}_{i}^{\mathrm{lo}}, \mathrm{st}_{i}^{u p}$. The transportation times $\mathrm{tsa}_{v i j}$ between each pair of ports $(i, j)$ are also given

The set of vessels $v \in V$ with upper bound load $\mathrm{la}_{v}^{\text {up }}$ are given. For each vessel, the route is given by the 0-1 parameter $\mathrm{x}_{v i j}$. Fixed durations for charge/discharge, $\mathrm{tfi}_{v i}$, and charge/discharge rate $\mathrm{rv}_{v i}$, are given. Vessels cannot wait at a port for longer than a time limit, TimeWUp $_{v}$. The time horizon, TH is given.

\section{Determine:}

The charge/discharge times and amounts of the vessels at ports are to be determined.

\section{Goal:}

Minimize the transportation, operation and waiting cost.

Consider the following illustrative problem and its solution with two vessels, one production port and two consumption ports. The profiles of the loads of the two vessels are depicted in Figures 2 and 3. As seen in Figure 2, V1 departs from the departure port $\mathrm{O}$ and arrives at the production port $\mathrm{P} 1$ at the beginning of the schedule period, and starts to charge. When a certain amount of product is charged, V1 departs from P1 for the first consumption port C1. After a certain time of sailing, V1 arrives at $\mathrm{C} 1$ and waits for operation, then discharges. After that, V1 sails to $C 2$, and discharges at C2 without waiting. Finally, V1 departs from C2 and sails to the destination port D. In Figure 3, a similar scenario for V2 is depicted. V2 visits P1 and $\mathrm{C} 2$ only.

In Figures 4 to 6 , the profiles of stocks of the three ports are depicted. For P1, the stock increases constantly at a certain rate, except when there is vessel charging. The stock will decrease rapidly when a vessel is charging at $\mathrm{P} 1$. For $\mathrm{C} 1$ and $\mathrm{C} 2$, the stocks decrease with certain rates except when there is vessel discharging. 


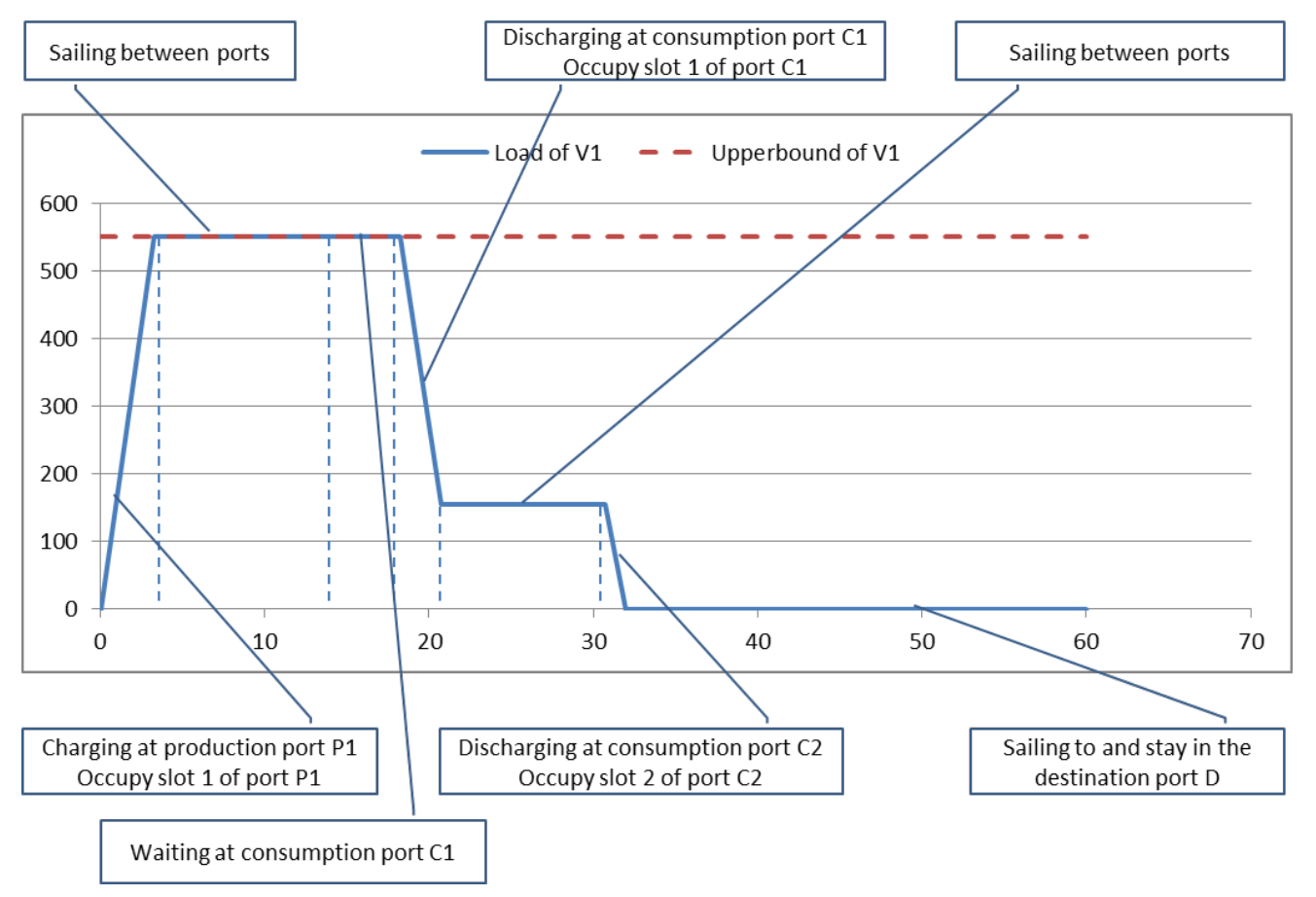

Figure 2 Load profile for vessel V1

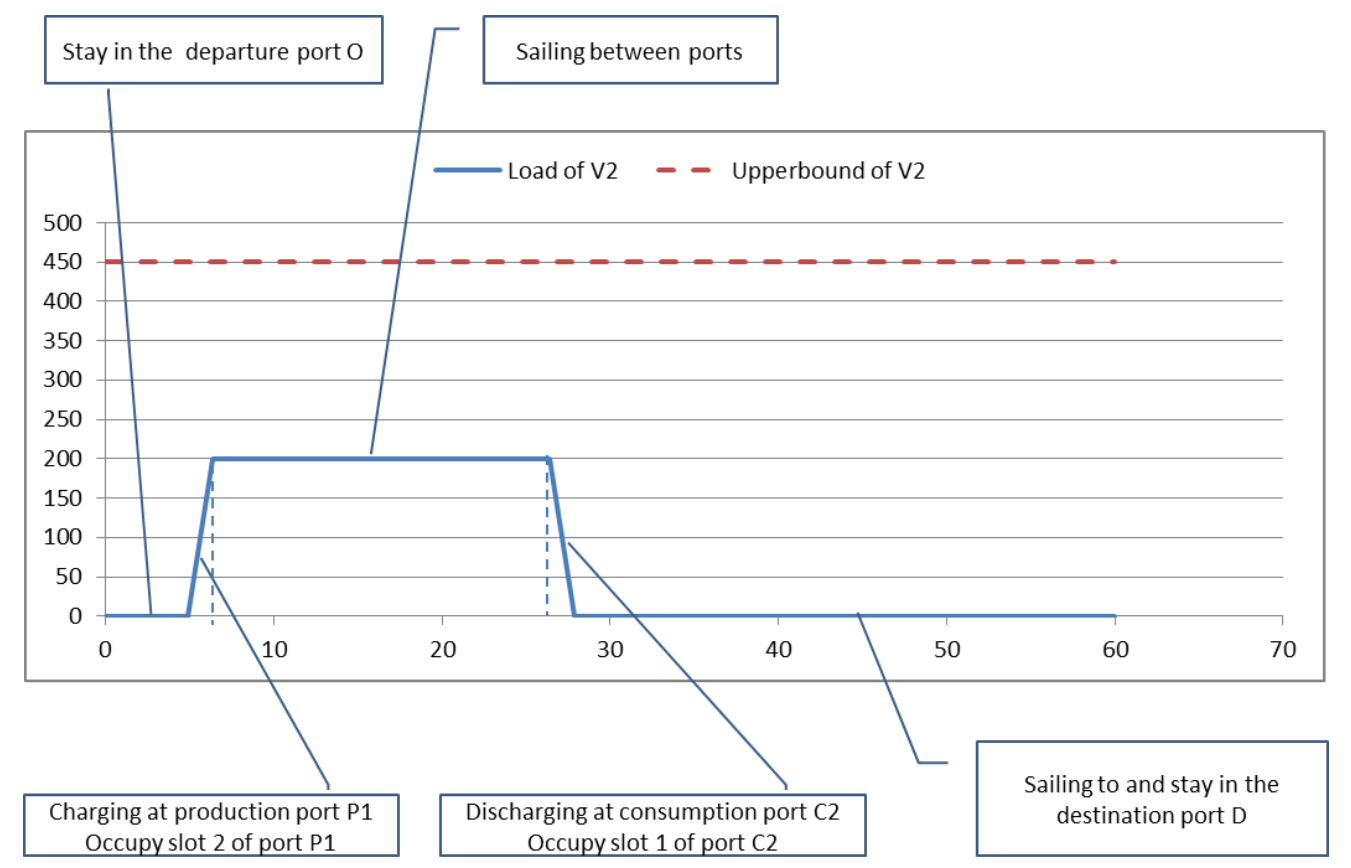

Figure 3 Load profile for vessel V2 


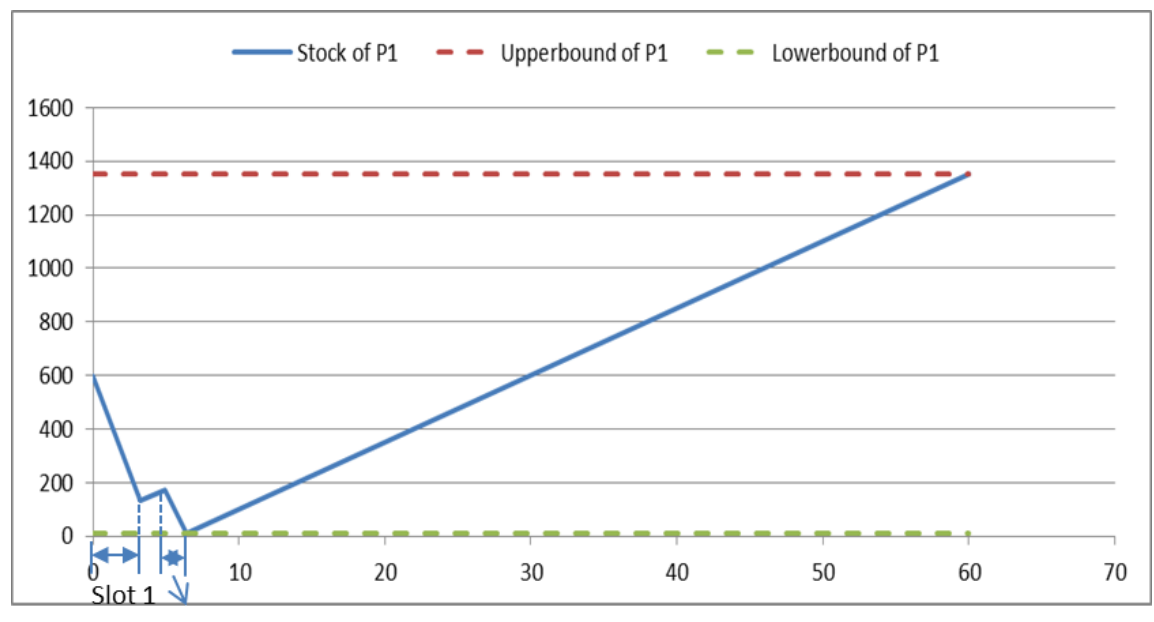

Slot 2

Figure 4 Inventory profile for production port P1

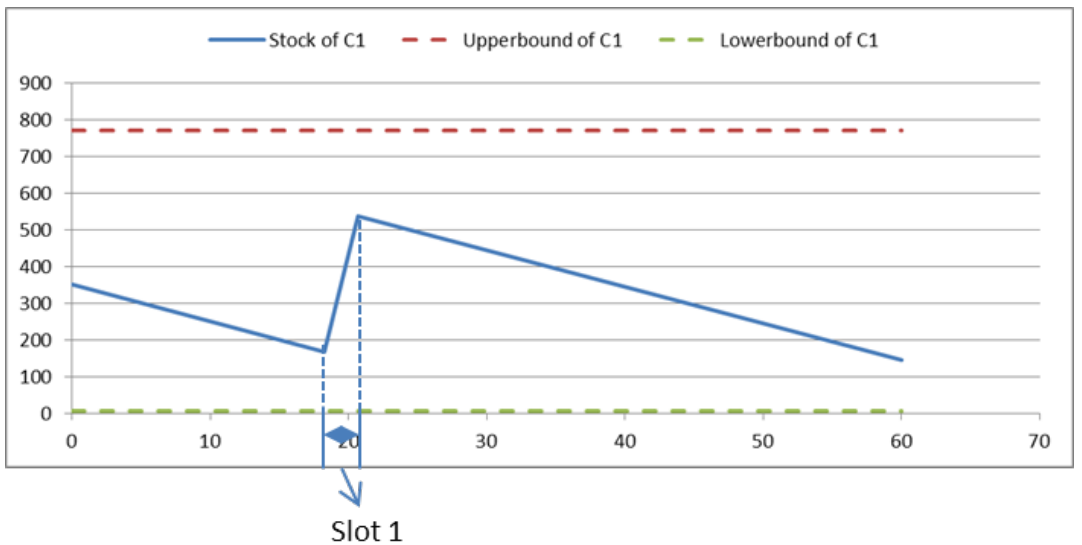

Figure 5 Inventory profile for consumption port C1

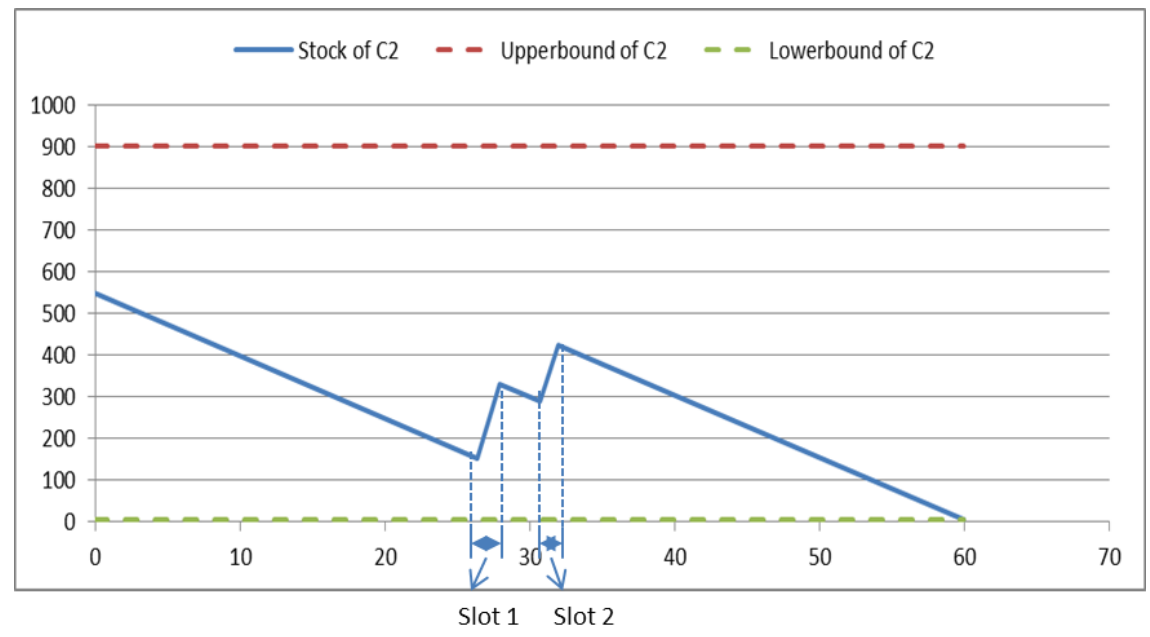

Figure 6 Inventory profile for consumption port C2

\section{Time representation}

For scheduling problems, there are two kinds of time representations, discrete time and 
continuous time representations. As pointed out by Floudas et al (2004), the continuous time representation can be classified into two groups, time slots and event points. We first describe the time representations before developing the models.

\section{a) Time slot representation}

Assuming first the case of single docks, we define time slots for each port. At every port, the operation of each vessel, including preparation to operate, is assigned a time slot as seen in Figure 7.

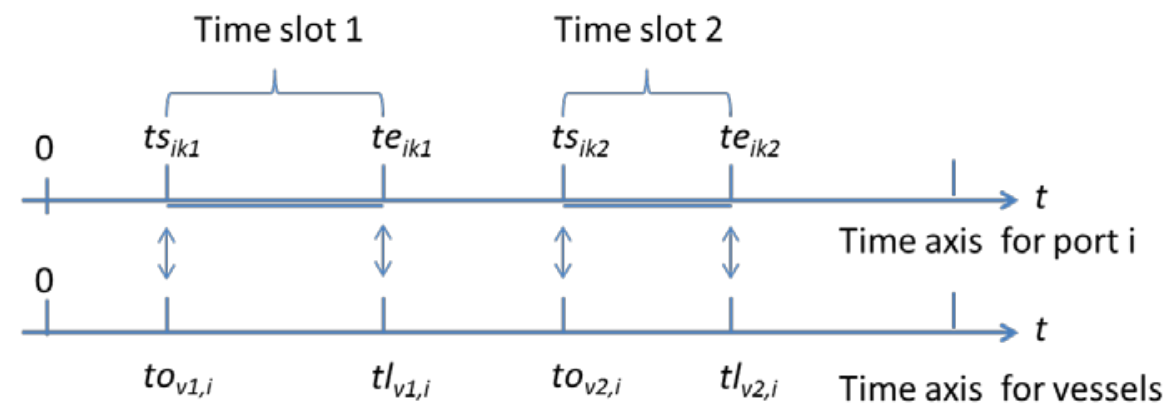

Figure 7 Time slot representation

For port $i$, time slots are defined with the start and end times as continuous variables. The vessels' continuous times for starting to prepare at and departing from the port (finishing operation) must match with the start and end times of a time slot, respectively. If there are $\mathrm{Num}_{i}$ vessels visiting port $i$, then $\mathrm{Num}_{i}$ time slots are defined for port $i$. In the illustrative problem, there are two vessels, V1 and V2, visiting ports $\mathrm{P} 1$ and $\mathrm{C} 2$, so two time slots, $\mathrm{k} 1$ and $\mathrm{k} 2$, are defined for $\mathrm{P} 1$ and $\mathrm{C} 2$, respectively, involving start times $t s_{i k}$ and end times $t e_{i k}$. For $\mathrm{C} 1$, only one time slot is defined. According to Figure 7, the start and end points of the two time slots are assigned to the two vessels respectively, with the start points assigned to the start to prepare times, and the end times to the departure times.

Consider now the time slots for the vessels. Recall the example illustrated in Figures 2-6. In Figure 2, vessel V1 visits all the three ports, so it is assigned three time slots (each slot corresponds to one port), and the three oblique line sections correspond to the slots. In Figure 3 , vessel V2 visits two ports, so it is assigned two time slots, and there are two oblique line sections. The horizontal sections indicate that the vessels are sailing in the sea, or staying in some port without operating. In Figures 4 and 6, there are two time slots. In Figure 6, the four turning points correspond to the two couples of start and end points. The actual start points are a bit earlier than the corresponding turning points because there are preparation times for charge/discharge. But the difference cannot be seen clearly since the preparation time $(0.5$ day) is much smaller. In Figure 4, the end time of the first slot and start time of second slot coincides with each other. This means that the middle turning point corresponds to both the end time of first slot and start time of second slot. In Figure 5, since only one vessel visits Port C1, only one time slot is defined.

\section{b) Event point representation}

In order to be able to handle parallel docks, we define event points $T_{i k}$ for each port. Starting to prepare for operation, starting to operate and departing from the port (finishing operation) 
matches with different event points as seen in Figure 8. The number of the points is three times that of the vessels (e.g. six event points for two vessels). There are similar descriptions on the profiles of the ports stocks and the vessels loads as in the case of the time slots.

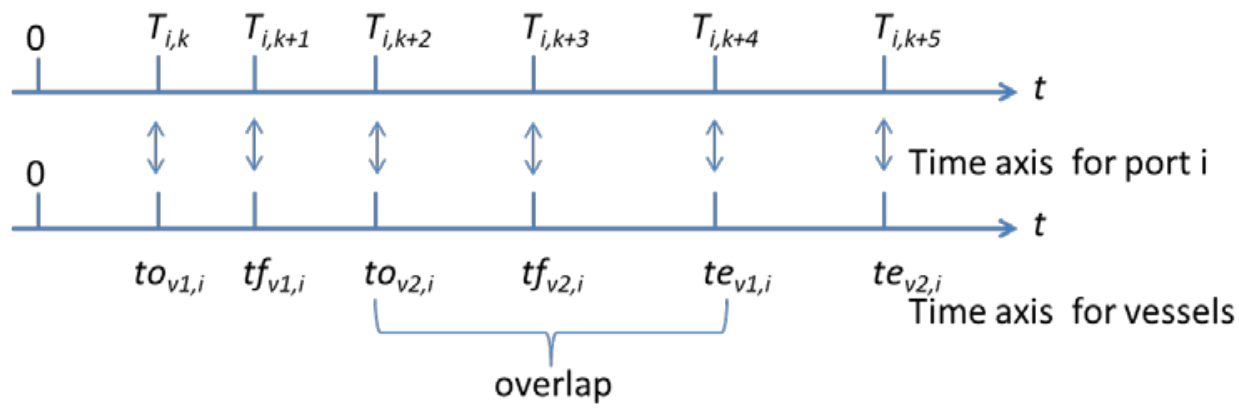

Figure 8 Event point representation

In Figure 8, the operation times (including the preparation time) of vessels V1 and V2 at port i overlap each other, that is, the two vessels operate simultaneously during time period $\left[\mathrm{to}_{\mathrm{v} 2, \mathrm{i}}, \mathrm{te}_{\mathrm{v} 1, \mathrm{i}}\right]$, since the time of starting to prepare for operation of vessel V2 is later than that of $\mathrm{V} 1$, and earlier than the time of finishing operation of $\mathrm{V} 1$. That is $\mathrm{to}_{\mathrm{v} 1, \mathrm{i}}<\mathrm{to}_{\mathrm{v} 2, \mathrm{i}}$ and $\mathrm{te}_{\mathrm{v} 1, \mathrm{i}}>$ $\mathrm{to}_{\mathrm{v} 1, \mathrm{i}}$. Hence, berth capacity of port i can be greater than one.

c) Discrete time representation

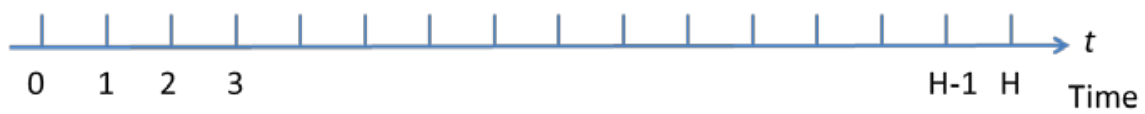

Figure 9 Discrete time representation

For the discrete time representation, we discretize the time horizon into a number of uniform time intervals (Figure 9). All the vessels and ports use the same time axis. Because all the events (departure, arrival, start and finish preparing, start and finish operation) occur at the fixed time intervals, the greatest common factor (GCF) of the preparation times and sailing times is adopted as the size of time intervals.

\section{Scheduling models}

\subsection{Continuous time model based on time slots}




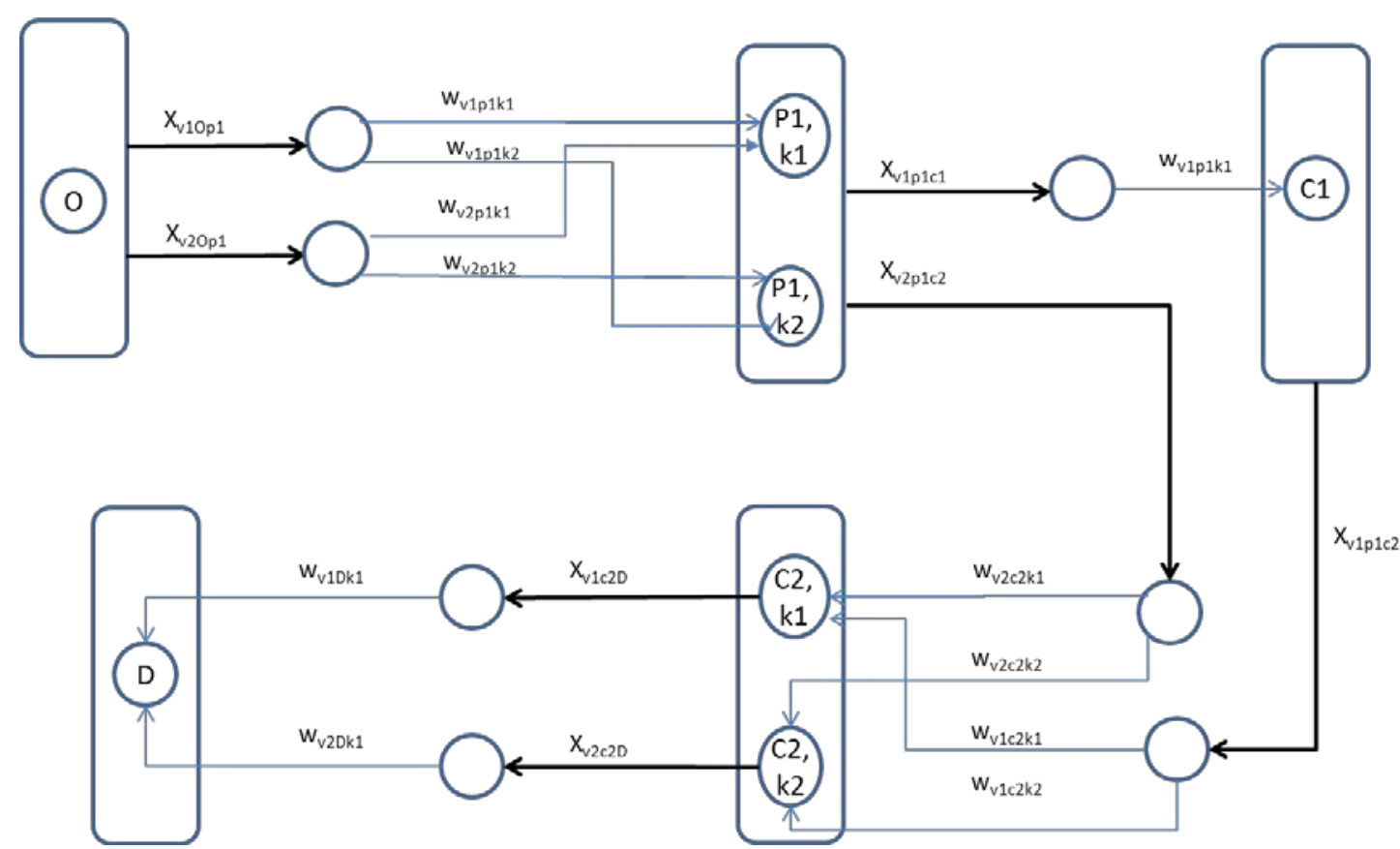

Figure 10 Sketch of the assignment of the operations to the corresponding ports

The basic idea of the time slot based model is to assign the operations to the corresponding ports. Figure 10 illustrates the basic idea, where $\mathrm{x}_{\mathrm{vij}}$ is a 0-1 parameter indicating whether vessel $v$ visits port $i$ and $j$ in succession. The $0-1$ binary variables $w_{v i k}$ are defined to denote whether vessel $v$ visits port $i$ at time slot $k$. If a vessel arrives at a port from another port, a time slot is assigned to it, and each slot can be assigned to it, but a slot can only be assigned to no more than one vessel, and no more than one slot can be assigned to one vessel. Then, we have the following constraints.

\section{Time slot constraints}

The subsequent slot cannot start before the preceding one ends, and the end time of a slot $k$ of port $i$ is later than the start time.

$$
\begin{aligned}
& t s_{i k+1} \geq t e_{i k}, \forall k \in K_{i} \backslash k_{i m}, i \in N^{p} \cup N^{c} \cup O \cup D \\
& t e_{i k} \geq t s_{i k}, \forall k \in K_{i}, i \in N^{p} \cup N^{c} \cup O \cup D
\end{aligned}
$$

where $k_{i m}$ indicates the last time slot of port $i$.

\section{Assignment constraints}

If vessel $v$ visits port $j$, a time slot of port $j$ is assigned to vessel $v$. Except for the departure port, vessel $v$ visits port $j$ means that it must come from a preceding port.

$$
\sum_{k \in K_{j}} w_{v j k}=\sum_{i \in N^{p} \cup N^{c} \cup o} \mathrm{x}_{v i j}, \forall v \in V, j \in N^{p} \cup N^{c} \cup D
$$

No more than one vessel can operate at any port at the same time.

$$
\sum_{v \in V} w_{v i k} \leq 1, \forall k \in K_{i}, i \in N^{p} \cup N^{c} \cup O \cup D
$$

If a time slot is assigned to a vessel, it means that its start time to prepare for operation at and departing time from the port are forced to the start and end time of the slot, respectively. Otherwise, if the time slot is not assigned to a vessel, the constraints are ignored.

$$
\left[\begin{array}{c}
w_{v i k} \\
t o_{v i}=t s_{i k} \\
t l_{v i}=t e_{i k}
\end{array}\right] \vee\left[\neg w_{v i k}\right], \forall v \in \mathrm{V}, k \in K_{i}, i \in N^{p} \cup N^{c} \cup O \cup D
$$

We usually reformulate the disjunction in (5) into the following big-M inequalities: 


$$
\begin{aligned}
& -\mathrm{M}\left(1-w_{v i k}\right) \leq t o_{v i}-t s_{i k} \leq M\left(1-w_{v i k}\right), \\
& \forall v \in V, k \in K_{i}, i \in N^{p} \cup N^{c} \cup O \cup D \\
& -\mathrm{M}\left(1-w_{v i k}\right) \leq t l_{v i}-t e_{i k} \leq M\left(1-w_{v i k}\right), \\
& \forall v \in V, k \in K_{i}, i \in N^{p} \cup N^{c} \cup O \cup D
\end{aligned}
$$

\section{Operational time constraints}

If a vessel departs from port $i$ to port $j$, its arrival time at $j$ equals to its departure time at $i$ plus the sailing time.

$$
t a_{v j}=\sum_{i \in N^{p} \cup N^{c}} \mathrm{x}_{v i j}\left(t l_{v i}+\mathrm{tsa}_{v i j}\right), \forall v \in V, j \in N^{p} \cup N^{c} \cup D
$$

A vessel can only start to prepare for charge/discharge operation after it arrives at a port.

$$
t o_{v i} \geq t a_{v i}, \forall v \in V, i \in N^{p} \cup N^{c} \cup O \cup D
$$

If $\mathrm{x}_{\mathrm{vij}}=0$, equality holds for an optimal solution because waiting incurs in a cost.

The interval between the arrival time and the start time to prepare for operation is the waiting time.

$$
t w_{v i}=t o_{v i}-t a_{v i}, \forall v \in V, i \in N^{p} \cup N^{c} \cup O \cup D
$$

If a vessel visits a port, it departs after a certain operation time, involving the preparation and variable operation time, which depends on the amount of charged/discharged product.

$$
t l_{v i}-t o_{v i}=\sum_{j \in N^{p} \cup N^{c}} \mathrm{x}_{v j i}\left(t f i_{v i}+q_{v i} / r v_{v i}\right), \forall v \in V, i \in N^{p} \cup N^{c}
$$

At the departure and destination ports, any vessel departs immediately after it operates.

$$
t l_{v i}-t o_{v i}=0, \forall v \in V, i \in O \cup D
$$

A vessel cannot wait longer than for a specified time limit.

$$
t w_{v i} \leq \text { TimeWUp }_{v}, \forall v \in V, i \in N^{p} \cup N^{c} \cup O \cup D
$$

Any vessel must depart from the destination port before the time horizon ends

$$
t l_{v d} \leq T H, \forall v \in V
$$

\section{Stock balance constraints}

A port's stock at the end of a slot equals to that at the beginning plus/minus the amount of production/consumption and that discharged/charged by the vessel.

$$
\begin{aligned}
& s t_{i k}^{s}-\sum_{v \in V}\left(w_{v i k} q_{v i}\right)+P_{i}\left(t e_{i k}-t s_{i k}\right)=s t_{i k}^{e}, \quad \forall k \in K_{i}, i \in N^{p} \\
& s t_{i k}^{s}+\sum_{v \in V}\left(w_{v i k} d_{v i}\right)-Q_{i}\left(t e_{i k}-t s_{i k}\right)=s t_{i k}^{e}, \forall k \in K_{i}, i \in N^{c}
\end{aligned}
$$

A port's stock at the beginning of the subsequent slot equals to that at the end of the preceding slot plus/minus the amount of production/consumption.

$$
\begin{aligned}
& s t_{i k}^{e}+P_{i}\left(t s_{i k+1}-t e_{i k}\right)=s t_{i k+1}^{s}, \forall k \in K_{i} \backslash k_{i m}, i \in N^{p} \\
& s t_{i k}^{e}-Q_{i}\left(t s_{i k+1}-t e_{i k}\right)=s t_{i k+1}^{s}, \forall k \in K_{i} \backslash k_{i m}, i \in N^{c}
\end{aligned}
$$

A port's stock at the beginning of the first time slot equals to the initial stock plus/minus the production/consumption amount.

$$
\begin{array}{ll}
s t_{i k 1}^{s}=s t_{i}^{0}+P_{i} t e_{i k 1}, & \forall i \in N^{p} \\
s t_{i k 1}^{s}=s t_{i}^{0}-Q_{i} t e_{i k 1}, & \forall i \in N^{c}
\end{array}
$$

where $k 1$ indicates the first time slot of port $i$.

A port's stock at the end of time horizon equals to that at the end of the last slot plus/minus the amount of production/consumption

$$
\begin{array}{ll}
s t_{i}^{f}=s t_{i k m}^{e}+P_{i}\left(\mathrm{TH}-t e_{i k m}\right), & \forall i \in N^{p} \\
s t_{i}^{f}=s t_{i k m}^{e}-Q_{i}\left(\mathrm{TH}-t e_{i k m}\right) & \forall i \in N^{c}
\end{array}
$$

The ports' stock must lie between lower and upper bounds.

$$
s t_{i}^{l o} \leq s t_{i}^{f} \leq s t_{i}^{u p}, \forall i \in N^{p} \cup N^{c}
$$




$$
\begin{aligned}
& s t_{i}^{l o} \leq s t_{i k}^{s} \leq s t_{i}^{u p}, \forall i \in N^{p} \cup N^{c}, k \in K_{i} \\
& s t_{i}^{l o} \leq s t_{i k}^{e} \leq s t_{i}^{u p}, \forall i \in N^{p} \cup N^{c}, k \in K_{i}
\end{aligned}
$$

\section{Load balance constraints}

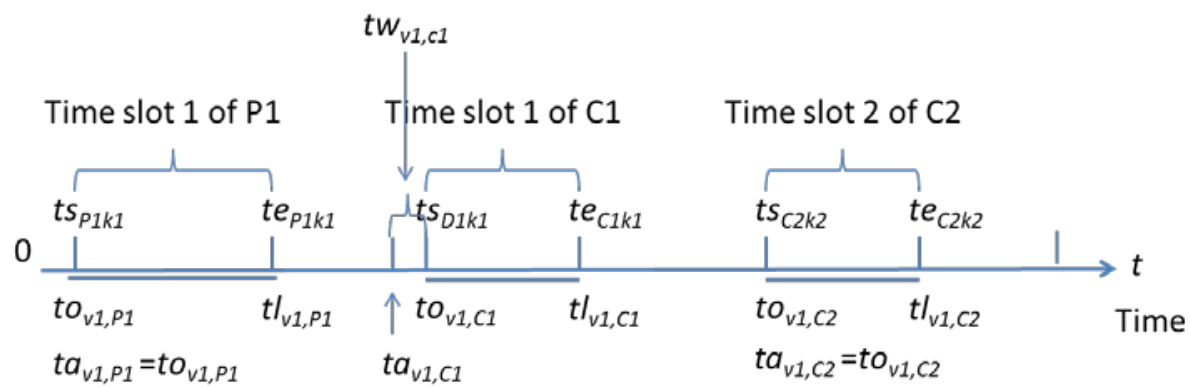

Figure 11 Sketch of vessel's load calculation

Generally, a vessel visits more than one port. When a vessel visits a port, its load changes according to the operations. Calculation of a vessel's load involves different ports as seen in Figure 11. The load of V1 increases after visiting P1, and decreases after visiting $\mathrm{C} 1$ and $\mathrm{C} 2$. The amounts of increase/decrease depend on the operation times.

If vessel $v$ visits ports $i$ and $j$ successively, its load before operation at port $j$ equals to that at port $i$ plus/minus the charge/discharge amounts at port $i$.

$$
\begin{aligned}
& \left(\sum_{i \in \in N^{p} \cup N^{c} \cup O} \mathrm{x}_{v i j} l a_{v i}+q_{v j}\right) \sum_{k \in K_{i}} w_{v j k}=l a_{v j}, \quad \forall v \in V, j \in N^{p} \cup D \\
& \left(\sum_{i \in \in N^{p} \cup N^{c} \cup O} \mathrm{x}_{v i j} l a_{v i}-d_{v j}\right) \sum_{k \in K_{i}} w_{v j k}=l a_{v j}, \forall v \in V, j \in N^{c} \cup D
\end{aligned}
$$

Since $l a_{v i}, q_{v j}, d_{v j}$ and $w_{v i k}$ are variables, constraints (26) and (27) are nonlinear. To linearize them, we introduce new continuous variables $w l a_{v i j k}, w l a 1_{v i j k}, w q_{v j k}, w q 1_{v j k}$ $w d_{v j k}, w d 1_{v j k}$, and new constraints as follows.

$$
\begin{aligned}
& w l a_{v i j k}+w_{l a} 1_{v i j k}=l a_{v i} \text {, } \\
& \forall v \in V, k \in K_{i}, i \in N^{p} \cup N^{c} \cup O, j \in N^{p} \cup N^{c} \cup D \\
& w l a_{v i j k} \leq l a_{v}^{u p} w_{v j k}, \forall v \in V, k \in K_{i} \text {, } \\
& i \in N^{p} \cup N^{c} \cup O, j \in N^{p} \cup N^{c} \cup D \\
& w l a 1_{v i j k} \leq l a_{v}^{u p}\left(1-w_{v j k}\right) \text {, } \\
& \forall v \in V, k \in K_{i}, i \in N^{p} \cup N^{c} \cup O, j \in N^{p} \cup N^{c} \cup D \\
& w q_{v j k}+w q 1_{v j k}=q_{v j}, \forall v \in V, k \in K_{i}, j \in N^{p} \cup D \\
& w q_{v j k} \leq q_{v j}^{u p} w_{v j k}, \forall v \in V, k \in K_{i}, j \in N^{p} \cup D \\
& w q 1_{v j k} \leq q_{v j}^{u p}\left(1-w_{v j k}\right), \forall v \in V, k \in K_{i}, j \in N^{p} \cup D \\
& w d_{v j k}+w d 1_{v j k}=d_{v j}, \forall v \in V, k \in K_{i}, j \in N^{c} \cup D \\
& w d_{v j k} \leq d_{v j}^{u p} w_{v j k}, \forall v \in V, k \in K_{i}, j \in N^{c} \cup D \\
& w d 1_{v j k} \leq d_{v j}^{u p}\left(1-w_{v k}\right), \forall v \in V, k \in K_{i}, j \in N^{c} \cup D
\end{aligned}
$$

The equations in (26) and (27) are then replaced by the new constraints

$\sum_{i \in \in N^{p} \cup N^{c} \cup o} \sum_{k \in K_{i}} \mathrm{x}_{v i j} w l a_{v i j k}+\sum_{k \in K} w q_{v j k}=l a_{v j}$,

$\forall \mathrm{v} \in V, j \in N^{p} \cup D$

$\sum_{i \in \in N^{p} \cup N^{c} \cup o} \sum_{k \in K_{i}} \mathrm{x}_{v i j} w l a_{v i j k}-\sum_{k \in K} w d_{v j k}=l a_{v j}$

$\forall \mathrm{v} \in V, j \in N^{c} \cup D$

Loads must lie between lower and upper bounds (the lower bound is generally zero).

$$
l a_{v i}^{l o} \leq l a_{v i} \leq l a_{v i}^{u p}, \forall v \in V, i \in N^{p} \cup N^{c} \cup D
$$

\section{Objective function}


The objective is to minimize the total cost $T C$, including the sailing cost (fixed sailing cost and variable sailing cost dependent on the load), waiting cost, and operation cost.

$$
\begin{array}{r}
T C=\sum_{v \in V, i, j \in N^{p} \cup N^{c} \cup O \cup D} \mathrm{x}_{v i j}\left(C s f_{v}+C s v_{v} l a_{v i}\right) \mathrm{tsa}_{v i j} \\
+\sum_{v \in V, i \in N^{p} \cup N^{c} \cup O \cup D} C w_{v i}\left(t o_{v i}-t a_{v i}\right) \\
+\sum_{v \in V, i \in N^{p} \cup N^{c} \cup O \cup D} C o_{v i}\left(t l_{v i}-t o_{v i}\right)
\end{array}
$$

where $C s f_{v}, C s v_{v}, C w_{v i}$ and $C o_{v i}$ are the corresponding cost coefficients. In this way, the continuous time model based on time slots is as follows:

S: minimize $T C$

Subject to (1)-(4), (6)-(25) and (28)-(33).

\subsection{Reformulation of the continuous time model based on time slot}

Pinto and Grossmann (1996) presented a reformulation method of the time slot assignment constraints of a short term scheduling problem of multistage batch plants, which is similar to the big-M inequalities in (6) and (7). Instead of using equations (5), the reformulations is as follows. First, equation (4) is reformulated as,

$$
\sum_{v \in V} w_{v i k}+y_{i k}=1, \forall k \in K_{i}, i \in N^{p} \cup N^{c} \cup O \cup D
$$

where $y_{i k}$ is an auxiliary continuous variable.

Define new variables $\gamma s_{i k}=t s_{i k} y_{i k}$ and $\gamma e_{i k}=t e_{i k} y_{i k}, \tau s_{v i k}=w_{v i k} t s_{i k}$ and $\tau e_{v i k}=w_{v i k} t e_{i k}$, to reformulate the disjunction in (5) in nonlinear form.

$$
\begin{aligned}
& w_{v i k}\left(t o_{v i}-t s_{i k}\right)=0, \forall k \in K_{i}, v \in \mathrm{V}, i \in N^{p} \cup N^{c} \cup O \cup D \\
& \Leftrightarrow w_{v i k} t o_{v i}=w_{v i k} t s_{i k}, \forall k \in K_{i}, v \in \mathrm{V}, i \in N^{p} \cup N^{c} \cup O \cup D \\
& w_{v i k}\left(t l_{v i}-t e_{i k}\right)=0, \forall k \in K_{i}, v \in \mathrm{V}, i \in N^{p} \cup N^{c} \cup O \cup D \\
& \Leftrightarrow w_{v i k} t l_{v i}=w_{v i k} t e_{i k}, \forall k \in K_{i}, v \in \mathrm{V}, i \in N^{p} \cup N^{c} \cup O \cup D
\end{aligned}
$$

Add equations (37) and (39) over the time slots $k \in K_{i}$.

$$
\begin{aligned}
& \sum_{k \in K_{i}} w_{v i k} t o_{v i}=\sum_{k \in K_{i}} \tau s_{v i k}, \forall v \in \mathrm{V}, i \in N^{p} \cup N^{c} \cup O \cup D \\
& \sum_{k \in K_{i}} w_{v i k} t l_{v i}=\sum_{k \in K_{i}} \tau e_{v i k}, \forall v \in \mathrm{V}, i \in N^{p} \cup N^{c} \cup O \cup D
\end{aligned}
$$

Recall $\sum_{k \in K_{i}} w_{v j k}=\sum_{i \in \mathrm{N}^{\mathrm{p}} \mathrm{NN}_{\mathrm{N}} \mathrm{UO}} \mathrm{x}_{\mathrm{vij}}$. Then,

$$
\begin{aligned}
& t o_{v j} \sum_{i \in N^{p} \cup N^{c} \cup O} x_{v i j}=\sum_{k \in K_{i}} \tau s_{v j k}, \forall v \in \mathrm{V}, j \in N^{p} \cup N^{c} \cup O \cup D \\
& t l_{v j} \sum_{i \in N^{p} \mathrm{UN}^{c} \cup O} x_{v i j}=\sum_{k \in K_{i}} \tau e_{v j k}, \forall v \in \mathrm{V}, j \in N^{p} \cup N^{c} \cup O \cup D
\end{aligned}
$$

Adding equations (37) and (39) over vessels $v \in \mathrm{V}$, yields,

$$
\begin{aligned}
& \sum_{v \in V} w_{v i k} t o_{v i}=\sum_{v \in \mathrm{V}} w_{v i k} t s_{i k}, \forall k \in K_{i}, i \in N^{p} \cup N^{c} \cup O \cup D \\
& \sum_{v \in \mathrm{V}} w_{v i k} t l_{v i}=\sum_{v \in \mathrm{V}} w_{v i k} t e_{i k}, \forall k \in K_{i}, i \in N^{p} \cup N^{c} \cup O \cup D
\end{aligned}
$$

Since $w_{v i k} t o_{v i}=w_{v i k} t s_{i k}$ and $w_{v i k} t l_{v i}=w_{v i k} t e_{i k}$

$$
\begin{aligned}
& \tau s_{v i k}=w_{v i k} t o_{i k}, \forall k \in K_{i}, v \in \mathrm{V}, i \in N^{p} \cup N^{c} \cup O \cup D \\
& \tau e_{v i k}=w_{v i k} t l_{i k}, \forall k \in K_{i}, v \in \mathrm{V}, i \in N^{p} \cup N^{c} \cup O \cup D
\end{aligned}
$$

then,

$$
\sum_{v \in \mathrm{V}} \tau s_{v i k}=t s_{i k} \sum_{v \in \mathrm{V}} w_{v i k}, \forall k \in K_{i}, i \in N^{p} \cup N^{c} \cup O \cup D
$$




$$
\sum_{v \in \mathrm{V}} \tau e_{v i k}=t e_{i k} \sum_{v \in \mathrm{V}} w_{v i k}, \forall k \in K_{i}, i \in N^{p} \cup N^{c} \cup O \cup D
$$

Also recall $\quad \sum_{v \in \mathrm{V}} w_{v i k}+y_{i k}=1$

$$
\begin{aligned}
& \sum_{v \in \mathrm{V}} \tau s_{v i k}=t s_{i k}\left(1-y_{i k}\right), \forall k \in K_{i}, i \in N^{p} \cup N^{c} \cup O \cup D \\
& \sum_{v \in \mathrm{V}} \tau e_{v i k}=t e_{i k}\left(1-y_{i k}\right), \forall k \in K_{i}, i \in N^{p} \cup N^{c} \cup O \cup D
\end{aligned}
$$

Defining $\gamma s_{i k}=t s_{i k} y_{i k}$ and $\gamma e_{i k}=t e_{i k} y_{i k}$, yields,

$$
\begin{aligned}
t s_{i k} & =\sum_{v \in \mathrm{V}, i \in N^{p} \cup N^{c} \cup O \cup D} \tau \mathrm{s}_{v i k}+\gamma s_{i k}, \forall k \in K_{i}, i \in N^{p} \cup N^{c} \cup O \cup D \\
t e_{i k} & =\sum_{v \in \mathrm{V}, i \in N^{p} \cup N^{c} \cup O \cup D} \tau \mathrm{e}_{v i k}+\gamma e_{i k}, \forall k \in K_{i}, i \in N^{p} \cup N^{c} \cup O \cup D
\end{aligned}
$$

Finally, considering the definition of $\tau s_{v i k}, \tau e_{v i k}, \gamma s_{i k}$ and $\gamma e_{i k}$, we have the following constraints:

$$
\begin{aligned}
& 0 \leq \tau s_{v i k} \leq w_{v i k} \mathrm{M}, \forall k \in K_{i}, v \in \mathrm{V}, i \in N^{p} \cup N^{c} \cup O \cup D \\
& 0 \leq \tau e_{v i k} \leq w_{v i k} \mathrm{M}, \forall k \in K_{i}, v \in \mathrm{V}, i \in N^{p} \cup N^{c} \cup O \cup D \\
& 0 \leq \gamma s_{i k} \leq y_{i k} \mathrm{M}, \forall k \in K_{i}, v \in \mathrm{V}, i \in N^{p} \cup N^{c} \cup O \cup D \\
& 0 \leq \gamma e_{i k} \leq y_{i k} \mathrm{M}, \forall k \in K_{i}, v \in \mathrm{V}, i \in N^{p} \cup N^{c} \cup O \cup D
\end{aligned}
$$

In this way, the disjunction in (5) is replaced by equations (42), (43), and (52)- (57).

Hence, the reformulation of the continuous time model based on time slot is as follows:

RS: minimize $f(x, y)$

Subject to (1)-(3), (8)-(25), (28)-(33), (42), (43) and (52)-(57).

\section{Number of variables and constraints of $\mathbf{S}$ and $\mathrm{RS}$}

Consider that $\sum_{v \in \mathrm{V}} w_{v i k} \leq 1$ in model $\mathrm{S}$ is reformulated as $\sum_{v \in \mathrm{V}} w_{v i k}+y_{i k}=1$ in model RS, in which new variables $y_{i k}$ are introduced in model RS. Consider variables $\gamma s_{i k}=t s_{i k} y_{i k}$ and $\gamma e_{i k}=t e_{i k} y_{i k}, \tau s_{v i k}=w_{v i k} t s_{i k}$ and $\tau e_{v i k}=w_{v i k} t e_{i k}$. By matching $\gamma s_{i k}$ and $t s_{i k}$ one by one, the numbers of them are the same. Similarly, the number of $\gamma e_{i k}$ and $t e_{i k}, \tau s_{v i k}$ and $t s_{i k}$, $\tau e_{v i k}$ and $t e_{i k}$ are the same as each other. All the other variables in models $\mathrm{S}$ and RS are the same. Therefore, the total number of continuous variables in model RS is larger that in model $\mathrm{S}$ by the number of $y_{i k}$ variables, while the number of binary variables in models $\mathrm{S}$ and RS are the same. Consider constraints (6) and (7) in model RS. The number is $4 *|\mathrm{~V}| * \sum_{i}\left|K_{i}\right|$, where $K_{i}$ indicates the set of time slots of port $i$. Consider constraint (4), the number is $\sum_{i}\left|K_{i}\right|$. The total number is $(4 *|\mathrm{~V}|+1) * \sum_{i}\left|K_{i}\right|$. Consider constraints (42) and (43) in model $\mathrm{S}$, the number is $2 *|\mathrm{~V}||\mathrm{I}|$. With constraints (52) and (53) in model RS, the number is $2 * \sum_{i}\left|K_{i}\right|$. Consider constraints (54)-(57) in model RS, the number is $2 * \sum_{i}\left|K_{i}\right||\mathrm{V}|+2 * \sum_{i}\left|K_{i}\right|$. Adding all the above, we have that the number of the special constraints in RS is $2 *|\mathrm{~V}||\mathrm{I}|+2 *$ $\sum_{i}\left|K_{i}\right|+2 *|\mathrm{~V}| * \sum_{i}\left|K_{i}\right|+2 * \sum_{i}\left|K_{i}\right|$. Compared with $(4 *|\mathrm{~V}|+1) * \sum_{i}\left|K_{i}\right|$, it depends on the parameters which is lager between the two numbers of the equations of $S$ and RS.

$\mathrm{S}$ and RS are both time slot based models, for which, if some of the slots overlap with each other, it means that there are parallel docks. However, for instance, the stock constraints (15) and (16) are not correct. Since it is difficult to develop the correct set of stock constraints, 
models $\mathrm{S}$ and RS can only be applied for cases when there are no parallel docks.

\subsection{Continuous time model based on event points}

To formulate the problem for parallel docks, we develop a continuous time model based on event points.

First, we define the following 0-1 binary variables as follows.

$y_{v i k}^{s}$, vessel $v$ starts to prepare at port $i$ at time point $T_{i k}$

$y_{v i k}^{f}$, vessel $v$ finishes preparation and starts to operate at port $i$ at time point $T_{i k}$

$y_{v i k}^{e}$, vessel $v$ finishes operation at port $i$ at time point $T_{i k}$

\section{Time point constraints}

Time points keep an increasing order.

$$
T_{i, k+1} \geq T_{i k}, \forall i \in N^{p} \cup N^{c} \cup O \cup D, k \in K_{i} \backslash k_{i m}
$$

where $k_{i m}$ indicates the last time point for port $i$.

\section{Assignment constraints}

Event points are assigned to different operation times as follows.

$\left[\begin{array}{c}y_{v i k}^{S} \\ T_{i k}=t o_{v i}\end{array}\right] \vee\left[\neg y_{v i k}^{S}\right], \forall v \in V, k \in K_{i}, i \in N^{p} \cup N^{c} \cup O \cup D$

$\left[\begin{array}{c}y_{v i k}^{f} \\ T_{i k}=t f_{v i}\end{array}\right] \vee\left[\neg y_{v i k}^{f}\right], \forall v \in V, k \in K_{i}, i \in N^{p} \cup N^{c} \cup O \cup D$

$\left[\begin{array}{c}y_{v i k}^{e} \\ T_{i k}=t e_{v i}\end{array}\right] \vee\left[\neg y_{v i k}^{e}\right], \forall v \in V, k \in K_{i}, i \in N^{p} \cup N^{c} \cup O \cup D$

Equations (59)-(61) state that if an event point is assigned to an operation, it equals to the corresponding variable.

We reformulate equations (59)-(61) using big-M constraints,

$-\mathrm{M}\left(1-y_{v i k}^{S}\right) \leq T_{i k}-t o_{v i} \leq M\left(1-y_{v i k}^{S}\right), \forall v \in V, k \in K_{i}, i \in N^{p} \cup N^{c} \cup O \cup D$

$-\mathrm{M}\left(1-y_{v i k}^{f}\right) \leq T_{i k}-t f_{v i} \leq M\left(1-y_{v i k}^{f}\right), \forall v \in V, k \in K_{i}, i \in N^{p} \cup N^{c} \cup O \cup D$

$-\mathrm{M}\left(1-y_{v i k}^{e}\right) \leq T_{i k}-t e_{v i} \leq M\left(1-y_{v i k}^{e}\right), \forall v \in V, k \in K_{i}, i \in N^{p} \cup N^{c} \cup O \cup D$

\section{Visiting constraints}

At any port and at any time, the number of vessels, which have started to prepare, and have not finished operation, cannot exceed its upper bound.

$$
\sum_{v \in V}\left(\sum_{k \in K_{i}} y_{v i k}^{s}-\sum_{k \in K_{i}} y_{v i k}^{e}\right) \leq \operatorname{Cap}_{i}, \forall i \in N^{p} \cup N^{c} \cup O \cup D
$$

where $\mathrm{Cap}_{i}$ indicates the berth capacity of port $i$.

Vessel $v$ at port i can only start and finish operation once if it visits that port.

$$
\sum_{k \in K_{i}} y_{v i k}^{s}=\sum_{k \in K_{i}} y_{v i k}^{e}=\sum_{j \in N^{p} \cup N^{c} \cup o} \mathrm{x}_{v j i}, \forall v \in V, i \in N^{p} \cup N^{c} \cup D
$$

Vessel $v$ at departure port can only start to prepare and finish operation once.

$$
\sum_{k \in K_{i}} y_{v o k}^{s}=\sum_{k \in K_{i}} y_{v o k}^{e}=1, \forall v \in V
$$

\section{Operation time constraints}


If a vessel departs from port $i$ to port $j$, its arrival time at $i$ equals to its departure time at $j$ plus the sailing time.

$$
t a_{v j}=\sum_{i \in N^{p} \cup N^{c} \cup o} \mathrm{x}_{v i j}\left(t l_{v i}+t s a_{v i j}\right), \forall v \in V, j \in N^{p} \cup N^{c} \cup D
$$

The interval between the arrival time and the starting to prepare time is the waiting time.

$$
t w_{v i}=t o_{v i}-t a_{v i}, \forall v \in V, i \in N^{p} \cup N^{c} \cup O \cup D
$$

If vessel $v$ visits port $i$, there is a fixed preparation time

$$
t f_{v i}-t o_{v i}=\sum_{j \in N^{p} \cup N^{c}} \mathrm{x}_{v j i} \mathrm{tfi}_{v i}, \forall v \in V, i \in N^{p} \cup N^{c}
$$

If vessel $v$ visits port $i$, the variable operation time depends on the charged/discharged amount.

$$
\begin{aligned}
& t l_{v i}-t f_{v i}=\sum_{j \in N^{p} \cup N^{c}} \mathrm{x}_{v j i} q_{v i} / r v_{v i}, \quad \forall v \in V, i \in N^{p} \\
& t l_{v i}-t f_{v i}=\sum_{j \in N^{p} \cup N^{c}} \mathrm{x}_{v j i} d_{v i} / r v_{v i}, \forall v \in V, i \in N^{c}
\end{aligned}
$$

At the departure and destination ports, vessel $v$ departs when it starts the operation of charge/discharge.

$$
t l_{v i}-t o_{v i}=0, \forall v \in V, i \in O \cup D
$$

The waiting time cannot exceed its upper bound.

$$
t w_{v i} \leq \text { TimeWUp }_{v}, \forall v \in V, i \in N^{p} \cup N^{c} \cup O \cup D
$$

Vessels must depart from the destination port before the time horizon ends.

$$
t l_{v d} \leq T H, \forall v \in V
$$

\section{Stock balance constraints}

A port's stock at the first event point equals to the initial stock plus/minus the production/ consumption amount.

$$
\begin{aligned}
& s t_{i}^{0}+P_{i} T_{i k 1}=s t_{i k 1}, \quad \forall i \in N^{p} \\
& s t_{i}^{0}-Q_{i} T_{i k 1}=s t_{i k 1}, \forall i \in N^{c}
\end{aligned}
$$

The difference between the stocks of the successive event points is the summation of the production/consumption amount and the charged/discharged amount. If vessel $v$ finishes preparation and does not finish operation at port $i$, the product is charged/discharged at a certain rate.

$$
\begin{aligned}
& s t_{i k}-\sum_{v}\left(\sum_{k^{\prime} \leq k} y_{v i k^{\prime}}^{f}-\sum_{k^{\prime} \leq k} y_{v i k^{\prime}}^{e}\right)\left(T_{i, k+1}-T_{i k}\right) q_{v i} \\
& +P_{i}\left(T_{i, k+1}-T_{i k}\right)=s t_{i, k+1}, \forall k \in K_{i} \backslash k_{i m}, i \in N^{p} \\
& s t_{i k}-\sum_{v}\left(\sum_{k^{\prime} \leq k} y_{v i k^{\prime}}^{f}-\sum_{k^{\prime} \leq k} y_{v i k^{\prime}}^{e}\right)\left(T_{i, k+1}-T_{i k}\right) d_{v i} \\
& -Q_{i}\left(T_{i, k+1}-T_{i k}\right)=s t_{i, k+1}, \forall k \in K_{i} \backslash k_{i m}, i \in N^{c}
\end{aligned}
$$




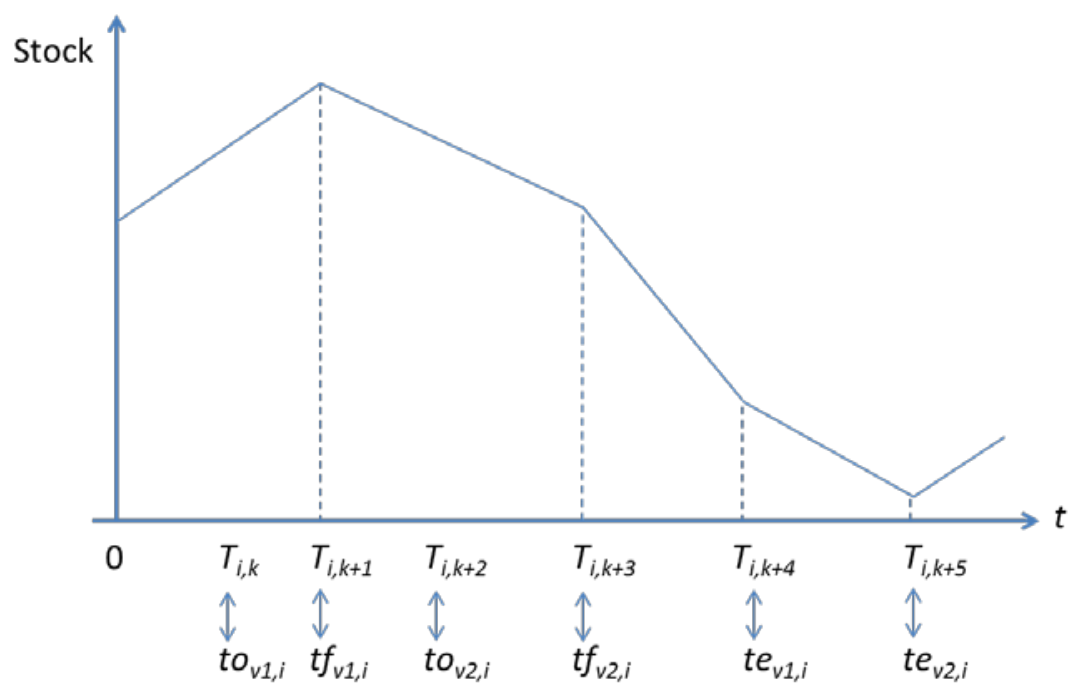

Figure 12 An illustrative profile of a production port

The stock at the end of the time horizon equals to that at the last point, plus/minus the production/consumption amount.

$$
\begin{aligned}
& s t_{i}^{f}=s t_{i k m}+P_{i}\left(T H-T_{i k m}\right), i \in N^{p} \\
& s t_{i}^{f}=s t_{i k m}-Q_{i}\left(T H-T_{i k m}\right), i \in N^{c}
\end{aligned}
$$

Stocks must lie between lower and upper bounds.

$$
\begin{aligned}
& s t_{i}^{l o} \leq s t_{i k} \leq s t_{i}^{u p}, \forall k \in K_{i}, i \in N^{p} \cup N^{c} \\
& s t_{i}^{l o} \leq s t_{i}^{f} \leq s t_{i}^{u p}, \forall i \in N^{p} \cup N^{c}
\end{aligned}
$$

\section{Load balance constraints}

If vessel $v$ visits port $i$ and $j$ successively, its load before operation at port $j$ equals to that at port $i$ plus/minus the charge/discharge amount at port $i$.

$$
\begin{aligned}
& \left(\sum_{i \in \in N^{p} \cup N^{c} \cup O} \mathrm{x}_{v i j} l a_{v i}+q_{v j}\right) \sum_{k \in K} y_{v i k}^{f}=l a_{v j}, \forall v \in V, j \in N^{p} \\
& \left(\sum_{i \in \in N^{p}{ }^{N^{c} \cup O}} \mathrm{x}_{v i j} l a_{v i}-d_{v j}\right) \sum_{k \in K} y_{v i k}^{f}=l a_{v j}, \forall v \in V, j \in N^{c}
\end{aligned}
$$

The charge/discharge time depends on the amount.

$$
\begin{aligned}
& t l_{v i}-t f_{v i}=\sum_{j \in N^{p} \cup N^{c}} \mathrm{x}_{v j i} q_{v i} / r v_{v i}, \quad \forall v \in V, i \in N^{p} \\
& t l_{v i}-t f_{v i}=\sum_{j \in N^{p} N^{c}} \mathrm{x}_{v j i} d_{v i} / r v_{v i}, \quad \forall v \in V, i \in N^{c}
\end{aligned}
$$

The vessel's load cannot exceed its lower and upper bounds

$$
l a_{v}^{l o} \leq l a_{v i} \leq l a_{v}^{u p}, \forall v \in V, i \in N^{p} \cup N^{c}
$$

\section{Objective function}

The objective function is the same as equation (34) in the time slot based models. Hence, the model is as follows:

E: minimize $T C$

Subject to (58), (62)-(88).

The event point based model can handle parallel docks. However, the size of the model becomes much larger due to a large number of 0-1 assignment binary variables. 


\subsection{Discrete time model}

Agra et al. (2013) developed a discrete time model for the maritime inventory routing problem. We present in this section a similar discrete time model, but in contrast to Agra et al. (2013) who consider only variable costs for operation and fixed costs for sailing, we consider fixed and variable costs for operation as well as fixed and variable costs for sailing. As shown below, the model corresponds to a fixed-charge network flow (FCNF) problem.

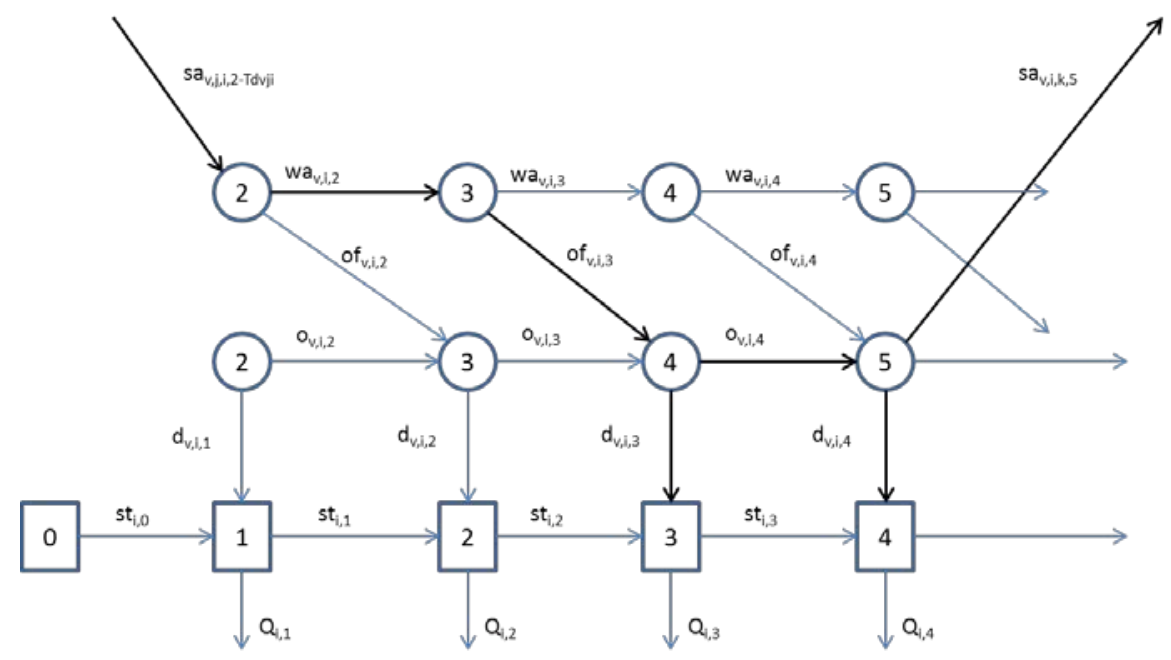

Figure 13 Operation at consumption port $i$ for vessel $v$ in the fixed charge flow network

In Figure 13, the squares indicate connections between the stocks at successive time points, and the discharged and consumed amounts in current time interval. The circles indicate the connections between the binary operation variables $o_{v i t}, o f_{v i t}, w a_{v i t}$, and $s a_{v i j t}$. We then have the following constraints.

\section{Routing constraints}

Vessel $v$ can only sail between ports $i$ and $j$ if there is a route planned from port $i$ to $j$ for vessel $v$.

$$
s a_{v i j t} \leq x_{v i j t}, \forall v \in \mathrm{V}, t \in T_{\text {dis }}, i, j \in N^{p} \cup N^{c} \cup O \cup D
$$

where $\mathrm{sa}_{\mathrm{vijt}}$ indicates that vessel $v$ is sailing between ports $\mathrm{i}$ and $\mathrm{j}$ at time $\mathrm{t}$.

The number of $s a_{v i j t}$ with value 1 for any $(v, i, j)$ equals to the total time vessel $v$ sailing between ports $i$ and $j$.

$$
\sum_{t \in T_{d i s}} s a_{v i j t}=T d_{v i j} * \mathrm{x}_{v i j t}, \forall v \in \mathrm{V}, i, j \in N^{p} \cup N^{c} \cup O \cup D
$$

where $T d_{v i j}$ is a parameter which indicates the transportation time between ports $i$ and $j$ for vessel $v$.

Any vessel must start and finish a schedule.

$$
\begin{aligned}
& \sum_{i \in O, j \in N^{p} \cup N^{c} \cup D} \sum_{t \in T_{d i s}} s a_{v i j t}=1 \quad \forall v \in V \\
& \sum_{i \in O N}{ }_{U N}{ }^{c} \cup, j \in D \\
& \sum_{t \in T_{d i s}} s a_{v i j t}=1 \quad \forall v \in V
\end{aligned}
$$

\section{Fixed charge network flow constraints}

A vessel cannot charge/discharge and prepare simultaneously.

$$
\sum_{v \in V}\left(o_{v i t}+o f_{v i t}\right) \leq 1, \forall i \in N^{p} \cup N^{c}, t \in T_{\text {dis }}
$$

Flow conservation I. Vessel $v$ departs or operates at time $t$ if it operates or prepares to operate at time $t-1$.

$$
o_{v i, t-1}+o f_{v i, t-1}=\sum_{j \in N^{p} \cup N^{c} \cup D} s a_{v i j t}+o_{v i t}, \forall i \in N^{p} \cup N^{c}, t \in T_{d i s} \backslash t_{1}
$$


Flow conservation II. Vessel $v$ waits or prepares for operation at time $t$ if it waits at time $t-1$, or departs from the preceding port at time $t-T d_{v i j}$.

$\sum_{j \in N^{p} \cup N^{c} \cup o} s a_{v j i, t-T d_{v i j}}+w a_{v i, t-1}=w a_{v i t}+o f_{v i t}$,

$\forall i \in N^{p} \cup N^{c}, t \in T_{\text {dis }} \backslash t_{1}, v \in \mathrm{V}$

Vessel $v$ cannot wait after operation.

$o_{v i, t-1}+o f_{v i, t-1} \leq o_{v i t}+o f_{v i t}+\sum_{j \in N^{p} \cup N^{c} \cup D} s a_{v i j t}$,

$\forall i \in N^{p} \cup N^{c}, t \in T_{d i s} \backslash t_{1}$

Vessel $v$ can only depart after operation.

$o_{v i, t-1} \geq \sum_{j \in N^{p} \cup N^{c} \cup D} s a_{v i j t}, \forall i \in N^{p} \cup N^{c}, t \in T_{d i s} \backslash t_{1}$

where $t_{1}$ is the first time point of the discrete time point.

Vessel $v$ can only operate after preparing to operate.

$$
\sum_{t 1 \geq t+1} o f_{v i, t 1}+o_{v i t} \leq 1, \forall i \in N^{p} \cup N^{c}, t \in T_{d i s} \backslash t_{1}
$$

\section{Stock balance constraints}

Stock of port $i$ at time $t+1$ equals to that at time $t$ minus/plus the charge/discharge amount plus/minus the production/consumption amount.

$$
\begin{aligned}
& s t_{i t}-\sum_{v \in V}\left(q r_{v i t} o_{v i t}\right)+P_{i}=s t_{i t+1}, \quad \forall i \in N^{p}, t \in T_{d i s} \backslash t_{m} \\
& s t_{i t}+\sum_{v \in V}\left(d r_{v i t} o_{v i t}\right)-Q_{i}=s t_{i t+1}, \forall i \in N^{c}, t \in T_{d i s} \backslash t_{m}
\end{aligned}
$$

where $t_{m}$ is the last time point of the discrete time point.

Any stock must lie between specified lower and upper bounds.

$$
s t_{i}^{l o} \leq s t_{i t} \leq s t_{i}^{u p}, \forall i \in N^{p} \cup N^{c}, t \in T_{\text {dis }}
$$

\section{Load balance constraints}

Load of vessel $v$ at time $t+1$ equals to that at time $t$ plus the charge amount minus the discharge amount.

$$
l a_{v t}+\sum_{i \in N^{p}} q r_{v i t}-\sum_{i \in N^{c}} d r_{v i t}=l a_{v t+1}, \forall v \in \mathrm{V}, t \in T_{d i s} \backslash t_{m}
$$

Load of any vessel at any time must lie between lower and upper bounds.

$$
l a_{v}^{l o} \leq l a_{v t} \leq l a_{v}^{u p}, \forall v \in \mathrm{V}, t \in T_{\text {dis }}
$$

\section{Upper bounds for waiting time}

The waiting time cannot exceed its upper bound

$$
\sum_{t \in T_{\text {dis }}} w_{\text {vit }} \leq \operatorname{TimeWU}_{v}, \forall i \in N^{p} \cup N^{c}
$$

\section{Preparation time for charging/discharging}

The number of of $\mathrm{vit}_{\text {wit }}$ with value 1 equals to the total preparation time

$$
\sum_{t \in T_{\text {dis }}} o f_{v i t}=t f_{v i}, \forall i \in N^{p} \cup N^{c}, v \in \mathrm{V}
$$

\section{Objective function}

The objective is to minimizing the total cost $T C^{\prime}$, including the sailing cost (fixed sailing cost and variable sailing cost dependent on the load), waiting cost, and operation cost.

$$
\begin{aligned}
T C^{\prime}= & \sum_{v i j t} s a_{v i j t}\left(C s f_{v}+C s v_{v} l a_{v t}\right)+\sum_{v i t} C w_{v i} w a_{v i t} \\
& +\sum_{v i t}\left(C o f_{v i} o f_{v i t}+C o_{v i} o_{v i t}\right)
\end{aligned}
$$

The discrete time model is as follows

D: minimize $T C^{\prime}$

Subject to (89)-(105). 
Even though the discrete time model is relatively straightforward to formulate, the size can be much larger because of a large number of potential event time interval assignments due to the time discretization. Furthermore, the quality of the solution will be worse if the time is discretized with longer intervals.

\section{Numerical results}

To test the four models, we considered multiple randomly generated instances of the six test | problems indicated in Table 1. Specifically, Tten feasible instances for each problem were selected among dozens of instances that were generated with random original stocks of the ports. Since the random generation does not always lead to feasible instances, only feasible ones are selected for each problem. We solve the different models on all the instances using CPLEX 12.5.0.0 within 10 hours limit of CPU time. In the discrete time models, the time is discretized uniformly with time intervals of 0.5 days. For the discrete time model as well as for the continuous time models we do not consider the addition of valid inequalities. While these have been developed for the discrete case (see Agra et al., 2013), at present none have been developed for the continuous case. Therefore, we have conducted the comparison without valid inequalities since our major goal is to establish the scope and potential of the continuous time models. The Aall-models are solved in GAMS 24.01 using a CPU Inter Xron E3110 $@ 3.0 \mathrm{GHz}$ with RAM 8.0Gb.

Table 1 Sizes of problems

\begin{tabular}{|l|l|l|l|l|l|l|}
\hline & $\begin{array}{l}\text { Problem } \\
1\end{array}$ & $\begin{array}{l}\text { Problem } \\
2\end{array}$ & $\begin{array}{l}\text { Problem } \\
3\end{array}$ & $\begin{array}{l}\text { Problem } \\
4\end{array}$ & Problem 5 & $\begin{array}{l}\text { Problem } \\
6\end{array}$ \\
\hline Number of production ports & 1 & 2 & 4 & 3 & 2 & 4 \\
\hline $\begin{array}{l}\text { Number of consumption } \\
\text { ports }\end{array}$ & 2 & 4 & 2 & 7 & 4 & 2 \\
\hline Number of vessels & 2 & 3 & 3 & 6 & 3 & 3 \\
\hline Berth capacity & 1 & 1 & 1 & 1 & 2 & 2 \\
\hline Time horizon/days & 60 & 70 & 70 & 80 & 70 & 70 \\
\hline
\end{tabular}

\subsection{Problem 1}

All the four models for problem 1 are solved for 10 random instances. The statistics and solutions are shown in Table 2.

Table 2 Statistics and solutions of different models for problem 1

\begin{tabular}{|l|l|l|l|l|}
\hline Model & \multicolumn{1}{|c|}{ S } & \multicolumn{1}{c|}{ RS } & \multicolumn{1}{c|}{ E } & \multicolumn{1}{c|}{ D } \\
\hline Number of constraints & 605 & 630 & 2,599 & 73,483 \\
\hline Number of variables & 478 & 546 & 1,613 & 53,041 \\
\hline Number of binary variables & 18 & 18 & 162 & 21,120 \\
\hline Average optimal objective & 6903 & 6903 & 6963 & 7386 \\
\hline $\begin{array}{l}\text { Average optimal objective } \\
\text { of LP Relaxation }\end{array}$ & 6673 & 6673 & 1673 & 1850 \\
\hline Average CPU time/s & 0.026 & 0.025 & 55.779 & 12.163 \\
\hline Average number of nodes & 2.6 & 3 & 14491 & 3987 \\
\hline
\end{tabular}


From Table 2, we can see that the size of model $E$ is much larger than those of models $S$ and RS, and that of model D is much larger than that of model E. As a result, the CPU times required by models $\mathrm{S}$ and RS are quite small, and those of models $\mathrm{E}$ and $\mathrm{D}$ are much longer. The optimal objective of model $\mathrm{D}$ is $7.1 \%$ higher than that of model $\mathrm{E}$, because the time domain is discretized. And as seen in Table 2, models $\mathrm{S}$ and RS are tighter than models $E$ and $D$. The schedule obtained by model $S$ for one of the instances of Problem 1 is illustrative in Figure 12.

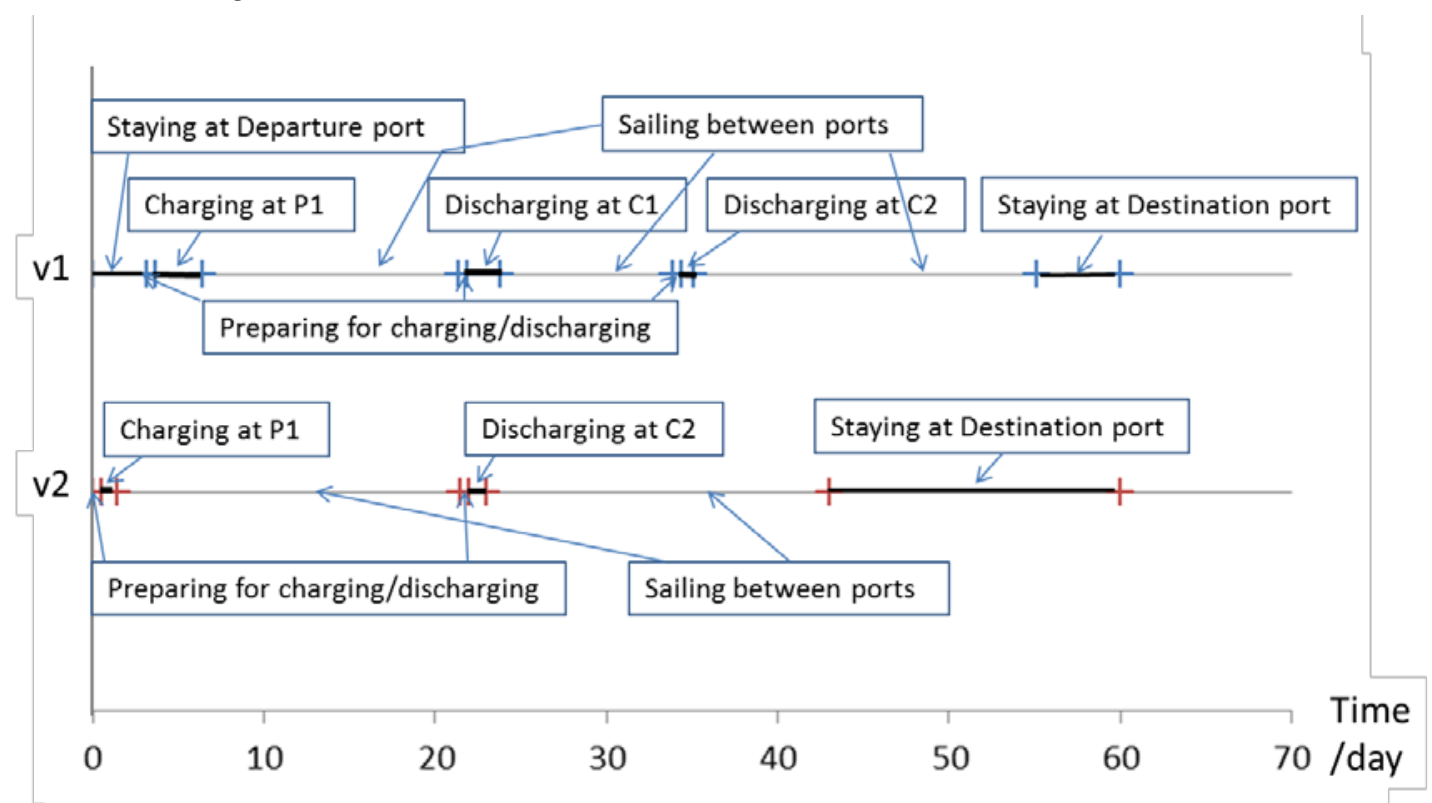

Figure 12 Gantt chart of model S for problem 1

\subsection{Problem 2}

All the four models for problem 2 are solved for 10 random instances. The statistics and solutions are shown in Table 3.

Table 3 Statistics and solutions of different models for problem 2

\begin{tabular}{|l|l|l|l|l|}
\hline Model & \multicolumn{1}{|c|}{ S } & \multicolumn{1}{c|}{ RS } & \multicolumn{1}{c|}{ E } & \multicolumn{1}{c|}{ D } \\
\hline Number of constraints & 1,281 & 1,308 & 7,003 & 126,621 \\
\hline Number of variables & 878 & 1,021 & 4,399 & 92,261 \\
\hline Number of binary variables & 45 & 45 & 405 & 36,960 \\
\hline \hline Average optimal objective & 12818 & 12818 & 12818 & 14755 \\
\hline $\begin{array}{l}\text { Average optimal objective } \\
\text { of LP Relaxation }\end{array}$ & 12054 & 12054 & 2721 & 3108 \\
\hline Average CPU time/s & 0.046 & 0.043 & 1144 & 11952 \\
\hline Average number of nodes & 12.2 & 4.1 & 151738 & 3591519 \\
\hline
\end{tabular}

There are similar trends as in problem 1. The number of nodes for model RS is one third as that of model S. This means RS performs better than S. The required CPU time of model D is ten times as long as that of model E. The optimal objective of D is $15.1 \%$ higher than the ones of the other models. 


\subsection{Problem 3}

All the four models for problem 3 are solved for 10 random instances. The statistics and solutions are shown in Table 4.

Table 4 Statistics and solutions of different models for problem 3

\begin{tabular}{|l|l|l|l|l|}
\hline Model & \multicolumn{1}{|c|}{ S } & \multicolumn{1}{c|}{ RS } & \multicolumn{1}{c|}{ E } & \multicolumn{1}{c|}{ D } \\
\hline Number of constraints & 1,287 & 1,314 & 7,009 & 126,621 \\
\hline Number of variables & 878 & 1,021 & 4,399 & 92,261 \\
\hline Number of binary variables & 45 & 45 & 405 & 36,960 \\
\hline Average optimal objective & 8934 & 8934 & 8944 & 11081 \\
\hline $\begin{array}{l}\text { Average optimal objective } \\
\text { of LP Relaxation }\end{array}$ & 7891 & 7891 & 2717 & 3046 \\
\hline Average CPU time/s & 0.055 & 0.045 & 4053 & 9278 \\
\hline Average number of nodes & 18.1 & 10 & 507901 & 2502848 \\
\hline
\end{tabular}

For problem 3 , there are similar trends as in problem 2. It should be noted is that the CPU time required by model $\mathrm{D}$ is more than twice as large as that of model $\mathrm{E}$, while the optimal objective of model D is $23.9 \%$ higher than that of model $\mathrm{E}$, and one of the instances for model $\mathrm{D}$ is infeasible due to time discretization. Also, the CPU time for models $\mathrm{S}$ and RS are several orders of magnitude smaller.

\subsection{Problem 4}

All the 4 models on problem 4 are solved For 10 random instances. The statistics and solutions are shown in Table 5.

Table 5 Statistics and solutions of different models for problem 4

\begin{tabular}{|l|l|l|l|l|}
\hline Model & \multicolumn{1}{|c|}{ S } & \multicolumn{1}{c|}{ RS } & \multicolumn{1}{c|}{ E } & \multicolumn{1}{c|}{ D } \\
\hline Number of constraints & 6,382 & 6,290 & 43,188 & 613,927 \\
\hline Number of variables & 4,097 & 4,589 & 27,811 & 452,161 \\
\hline Number of binary variables & 192 & 192 & 1,728 & 172,800 \\
\hline Optimal objective & 15826 & 15826 & $\begin{array}{l}\text { No feasible } \\
\text { Solutions }\end{array}$ & $\begin{array}{l}\text { No feasible } \\
\text { solutions }\end{array}$ \\
\hline $\begin{array}{l}\text { Average optimal objective } \\
\text { of LP Relaxation }\end{array}$ & 8189 & 8189 & 2901 & 3482 \\
\hline CPU time/s & 0.311 & 0.201 & 360000 & 360000 \\
\hline Number of nodes & 141.8 & 20.8 & & \\
\hline
\end{tabular}

Problem 4 is a larger scale problem. Note that models E and D cannot be solved within the time limit of 360000 seconds (100 hours), and no feasible solutions can be found with that time limit. In Table 5, we can see that models $S$ and RS are very efficient, with RS being faster than $S$.

\subsection{Problems 5 and 6}

Problems 5 and 6 involve parallel docks. Therefore, models $S$ and RS do not apply. Tables 6 and 7 show the statistics and solutions of models $E$ and $D$ for 10 random instances. The 
results show that model $\mathrm{D}$ takes longer time than model $\mathrm{E}$ for problem 5 , while model $\mathrm{E}$ takes longer time than model $\mathrm{D}$ for problem 6 . In both cases model $\mathrm{E}$ obtains a lower cost objective than model $\mathrm{D}$. The schedule of one of the instances of problem 5 is shown in Figure 13.

Table 6 Statistics and solutions of different models for problem 5 with 2 parallel docks

\begin{tabular}{|l|l|l|}
\hline Model & \multicolumn{1}{|c|}{ E } & \multicolumn{1}{c|}{ D } \\
\hline Number of constraints & 7,003 & 126,621 \\
\hline Number of variables & 4,399 & 92,261 \\
\hline Number of binary variables & 405 & 36,960 \\
\hline \hline Average optimal objective & 12475 & 14546 \\
\hline $\begin{array}{l}\text { Average optimal objective } \\
\text { of LP Relaxation }\end{array}$ & 2721 & 3102 \\
\hline Average CPU time/s & 15084 & 23088 \\
\hline Average number of nodes & 2473794 & 6615928 \\
\hline
\end{tabular}

Table 7 Statistics and solutions of different models for problem 6 with 2 parallel docks

\begin{tabular}{|l|l|l|}
\hline Model & \multicolumn{1}{|c|}{ E } & \multicolumn{1}{c|}{ D } \\
\hline Number of constraints & 7,009 & 126,621 \\
\hline Number of variables & 4,399 & 92,261 \\
\hline Number of binary variables & 405 & 36,960 \\
\hline Average optimal objective & 9011 & 11164 \\
\hline $\begin{array}{l}\text { Average optimal objective } \\
\text { of LP Relaxation }\end{array}$ & 2717 & 3049 \\
\hline Average CPU time/s & 17452 & 8556 \\
\hline Average number of nodes & 2435492 & 2422197 \\
\hline
\end{tabular}

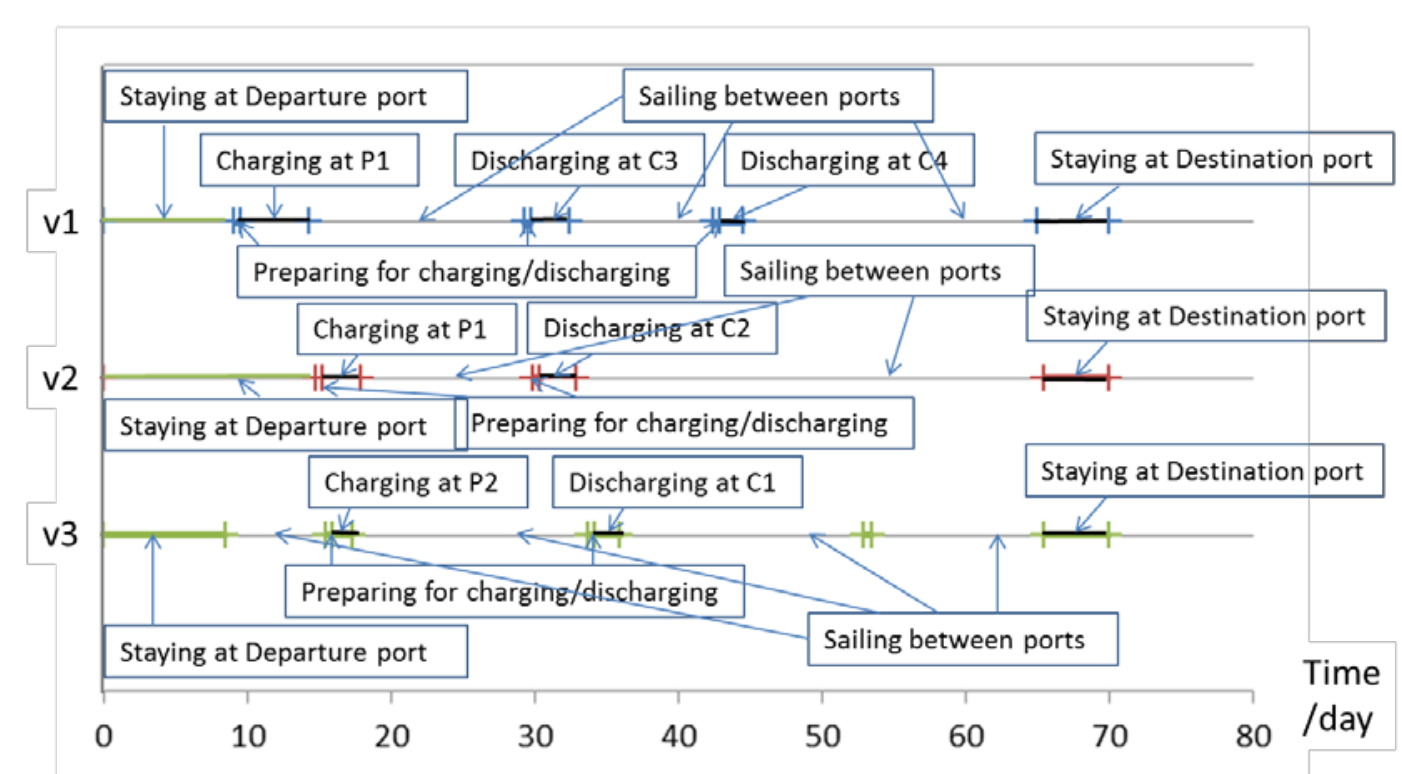

Figure 13 Gantt chart of problem 5 
From all the above results, we can conclude that for single dock problems the time slot models $\mathrm{S}$ and RS are solved much faster than event point model E and discrete time model_D, and RS performs best for larger instances. For the cases with parallel docks, only models $\mathrm{E}$ and D apply, with the continuous time models $\mathrm{E}$ obtaining significantly better objective function values than the discrete time model $\mathrm{D}$. On the other hand, the CPU time required depends on the particular instances.

\section{Conclusions}

A single product maritime inventory routing problem has been addressed in this paper. Three continuous time models and one discrete time model were presented. The first continuous time model S_S is based on time slots. By reformulating the slot assignment constraints, we obtained a second continuous model RSR. The statistics and solutions of the problems have shown that the reformulation can improve the computational efficiency. However, the two time slot based models cannot handle parallel docks. Therefore, we developed the third continuous time model EE, an event point based model, which can deal with parallel docks but introduces a large number of 0-1 binary variables. A discrete time model DD based on fixed-charge network flow was also developed that can deal with single/parallel docks. From the numerical results, the discrete time model DD was shown to lead to higher objective values than the continuous time event point based models since the latter do not involve an approximation of the timing of the events.

In summary, for the maritime inventory model, the computational results have shown that continuous time models have the potential of being more efficient than discrete time models. The time slot based models can be solved effectively for the problem with single docks. However, for large sized problems with parallel docks, even the continuous time event point model can require long CPU times. Hence, valid inequalities and/or decomposition algorithms may be required to effectively solve these problems.

\section{Acknowledgments}

The authors gratefully acknowledge financial support from the National Natural Science Foundation of China (No. 61273039) and from the Center of Advanced Process Decision-making at Carnegie Mellon University.

\section{Nomenclature}

Sets

$D \quad$ Set of destination ports containing 1 element

$N^{c} \quad$ Set of consumption ports with indices $i$ and $j$

$N^{p} \quad$ Set of production ports with indices $i$ and $j$

$0 \quad$ Set of departure ports containing 1 element

$V \quad$ Set of vessels with index $v$

$K_{i} \quad$ Set of time slots for port $i$ with index $k_{i}, k_{i 1}$ and $k_{i m}$ indicate the first and last slots, respectively..

$T_{i} \quad$ Set of event points for port $i$ with index $T_{i k}$ 
$T_{\text {dis }} \quad$ Set of time points for discrete time representation with index $t_{l}, t_{1}$ and $t_{m}$ indicate the first and last points, respectively.

\section{Binary variables}

$o_{\text {vit }} \quad 1$ if vessel $v$ operating at port $i$ in time period $t$

$o f_{\text {vit }} \quad 1$ if vessel $v$ preparing to operate at port $i$ in time period $t$

$s a_{v i j t} \quad 1$ if vessel $v$ sailing from $i$ to $j$ in time period $t$

$w_{\text {vik }} \quad 1$ if vessel $v$ visits port $i$ at time slot $k$

$w a_{v i t} \quad 1$ if vessel $v$ waiting at port $i$ in time period $t$

$y_{v i k}^{s} \quad 1$ if vessel $v$ starts to prepare at port $i$ at time point $T_{i k}$

$y_{v i k}^{f} \quad 1$ if vessel $v$ finishes preparation and starts to operate at port $i$ at time point $T_{i k}$

$y_{v i k}^{e} \quad 1$ if vessel $v$ finishes operation at port $i$ at time point $T_{i k}$

\section{Continuous variables}

$d_{v i} \quad$ Discharge amount of vessel $v$ at port $i$

$l a_{v i} \quad$ Load of vessel $v$ at port $i$ before operation

$q_{v i} \quad$ Charge amount of vessel $v$ at port $i$

$s t_{i t} \quad$ Stock of port $i$ at the beginning of time period $t$ for discrete time model

$s t_{i k}^{e} \quad$ Stock of port $i$ at the end of time slot $k$

$s t_{i}^{f} \quad$ Stock of port $i$ at the end of time horizon

$s t_{i k}^{S} \quad$ Stock of port $i$ at the beginning of time slot $k$

$t a_{v i} \quad$ Arrival time of vessel $v$ at port $i$

$t e_{i k} \quad$ End time of slot $k$ of port $i$

$t f_{v i} \quad$ Starting time of vessel $v$ to operate at port $i$

$t l_{v i} \quad$ Departure of time vessel $v$ from port $i$

$t o_{v i} \quad$ Starting time of vessel $v$ to prepare for operation at port $i$

$t s_{i k} \quad$ Starting time of slot $k$ of port $i$

$t w_{v i} \quad$ Time duration of vessel $v$ waits for at port $i$

$T_{i k} \quad$ the $k^{\text {th }}$ time point of port $i$

$T C, T C^{\prime} \quad$ Total cost

Parameters

$\mathrm{Cap}_{i}$

Berth capacity of port $i$

$\mathrm{Co}_{v \mathrm{i}}$

Operation cost of vessel $v$ at port $i$

$\mathrm{Csf}_{v} \quad$ Fixed cost of vessel $v$ for sailing

$\operatorname{Csv}_{v} \quad$ Coefficient of variable cost of vessel $v$ for sailing

$\mathrm{Cw}_{v i} \quad$ Coefficient of waiting cost of vessel $v$ at port $i$

$\mathrm{dr}_{v i t} \quad$ Discharge rate of vessel $v$ at port $i$ for discrete time model

$\operatorname{la}_{v}^{\text {lo }} \quad$ Lowerbound of vessel $v^{\prime}$ 's load

$\mathrm{la}_{v}^{\text {up }} \quad$ Upperbound of vessel $v^{\prime}$ 's load

$\mathrm{P}_{i} \quad$ Production rate of port $i$

$\mathrm{qr}_{v i t} \quad$ Charge rate of vessel $v$ at port $i$ for discrete time model

$\mathrm{Q}_{i} \quad$ Consumption rate of port $i$

$\mathrm{rv}_{v i} \quad$ Charge/discharge rate of vessel $v$ at port $i$

st $_{i}^{\text {to }} \quad$ Lowerbound of stock of port $i$ 


\begin{tabular}{|c|c|}
\hline $\mathrm{st}_{i}^{\mathrm{up}}$ & Upperbound of stock of port $i$ \\
\hline $\mathrm{Td}_{v i j}$ & Transportation time between port $i$ and $j$ for vessel $v$ for discrete time model \\
\hline $\mathrm{tfi}_{v i}$ & Preparation time for charging/discharging of vessel $v$ at port $i$ \\
\hline TH & Time horizon \\
\hline $\mathrm{TimeWUp}_{v}$ & Upperbound of vessel $v$ 's waiting time \\
\hline tsa $_{v i j}$ & $\begin{array}{l}\text { Transportation time between port } i \text { and } j \text { for vessel } v \text { for continuous time } \\
\text { model, in days }\end{array}$ \\
\hline$x_{v i j}$ & 1 indicates that vessel $v$ departs from $i$ to $j$ \\
\hline
\end{tabular}

\section{References}

Agra A., Andersson H., Christiansen M., Wolsey L. A maritime inventory routing problem: Discrete time formulations and valid inequalities. Networks, 2013, 62(4): $297-314$.

Al-Khayyal F., Hwang S.J.. Inventory constrained maritime routing and scheduling for multi-commodity liquid bulk, Part I: Applications and model, European Journal of Operational Research, 176 : 106-130, 2007.

Andersson H., Hoff A., Christiansen M., Hasle G., Løkketangen A. Industrial aspects and literature survey: Combined inventory management and routing. Computers \& Operations Research, 37: 1515-1536, 2010.

Bilgen B., Ozkarahan I. A mixed-integer linear programming model for bulk grain blending and shipping, International Journal of Production Economics, 107(2): 555-571, 2007.

Christiansen M., Fagerholt K., Ronen D.. Ship routing and scheduling: Status and perspectives. Transportation Science, 38: 1-18, 2004.

Christiansen M., Fagerholt K.. Maritime inventory routing problems. In: Floudas CA, Pardalos PM, editors, Encyclopedia of Optimization, second edition, Springer, 2009.

Floudas C. A., Lin X.. Continuous-time versus discrete-time approaches for scheduling of chemical processes: a review. Computers and Chemical Engineering, 28: 2109-2129, 2004.

Furman K.C., Song J.H., Kocis G.R., McDonald M.K., Warrick P.H.. Feedstock routing in the ExxonMobil downstream sector., Interfaces, 41 : 149-163, 2011.

Li J., Karimi I.A., Srinivasan R. Efficient bulk maritime logistics for the supply and delivery of multiple chemicals, Computers \& Chemical Engineering, 34(12): 2118 - 2128, 2010.

Pinto J. M., Grossmann I. E.. A Continuous Time Mixed Integer Linear Programming Model for Short Term Scheduling of Multistage Batch Plants. Industrial Engineering and Chemistry Research, 34: 3037-3051, 1996.

Ronen D. Cargo ships routing and scheduling: Survey of models and problems. European Journal of Operational Research, 12: 119-126, 1983.

Ronen D. Ship scheduling: The last decade. European Journal of Operational Research, 71(3): 325-333, 1993.

Savelsbergh M. W. P., Song J.-H. An optimization algorithm for the inventory routing problem with continuous moves. Computers \& Operations Research, 35: 2266-2282, 2008. 
Sherali H., Al-Yakoob, S. Hassan M. Fleet management models and algorithms for an oil-tanker routing and scheduling problem. IIE Transactions, 31: 395-406, 1999.

Sherali H., Al-Yakoob S. Determining an Optimal Fleet Mix and Schedules: Part I - Single Source and Destination, In J. Karloff editor, Integer Programming: Theory and Practice, 137-166, 2005.

Sherali H., Al-Yakoob S. Determining an Optimal Fleet Mix and Schedules: Part II - multiple sources and destinations, and the Option of Leasing Transshipment Depots, In J. Karloff editor, Integer Programming: Theory and Practice, 167-193, 2005.

Siswanto N., Essam D., Sarker R. Solving the ship inventory routing and scheduling problem with undedicated compartments. Computers \& Industrial Engineering, 2010 (DOI: 10.1016/j.cie.2010.06.011).

Song J.-H., Furman K.C.. A maritime inventory routing problem: Practical approach, Computers \& Operations Research, 2010 (DOI: 10.1016/j.cor.2010.10.031).

UNCTAD. Review of maritime transport. 2008. United Nations, New York and Geneva, 2008.

Vis, I. F. Koster A., R.. Transshipment of containers at a container terminal: An overview. European Journal of Operational Research, 147: 1-16, 2003. 